\title{
An Ellipsoidal Set-Membership Approach to Distributed Joint State and Sensor Fault Estimation of Autonomous Ground Vehicles
}

\author{
Eman Mousavinejad, Member, IEEE, Xiaohua Ge, Senior Member, IEEE, Qing-Long Han, Fellow, IEEE, \\ Teng Joon Lim, Fellow, IEEE, and Ljubo Vlacic, Senior Member, IEEE
}

\begin{abstract}
This paper is concerned with the problem of distributed joint state and sensor fault estimation for autonomous ground vehicles subject to unknown-but-bounded (UBB) external disturbance and measurement noise. In order to improve the estimation reliability and performance in cases of poor data collection and potential communication interruption, a multi-sensor network configuration is presented to cooperatively measure the vehicular yaw rate, and further compute local state and fault estimates. Toward this aim, an augmented descriptor vehicle model is first established, where the unknown sensor fault is modeled as an auxiliary state of the system model. Then, a new distributed ellipsoidal set-membership estimation approach is developed so as to construct an optimized bounding ellipsoidal set which guarantees to contain the vehicle's true state and the sensor fault at each time step despite the existence of UBB disturbance and measurement noises. Furthermore, a convex optimization algorithm is put forward such that the gain matrix of each distributed estimator can be recursively obtained. Finally, simulation results are provided to validate the effectiveness of the proposed approach.
\end{abstract}

Index Terms-Distributed set-membership estimation, autonomous ground vehicle (AGV), fault estimation, vehicle lateral dynamics, sensor networks.

\section{INTRODUCTION}

$\mathbf{R}$ ECENT advances in vehicle sensing and computing devices, intelligent driver-assistance systems, and wireless communication technologies during the last decade have considerably revitalized the development of autonomous ground vehicles (AGVs) [1] and autonomous surface vehicles [2]. As one of the components of intelligent transportation systems (ITS), AGVs with limited or no human intervention have been touted as a promising solution for improving vehicle safety and transportation efficiency, reducing traffic accidents, and providing an opportunity to conduct particular missions [3]. Moreover, advanced vehicular active safety technologies such as electronic stability program (ESP), active front steering

Corresponding author: Qing-Long Han.

E. Mousavinejad and T. J. Lim are with the School of Electrical and Information Engineering, The University of Sydney, Sydney, NSW 2006, Australia (emails: eman.mousavinejad@sydney.edu.au; tj.lim@sydney.edu.au). E. Mousavinejad is also with the School of Engineering and Built Environment, Griffith University, Gold Coast, QLD 4222, Australia (e-mail: eman.mousavinejad@griffith.edu.au).

X. Ge and Q.-L. Han are with the School of Software and Electrical Engineering, Swinburne University of Technology, Melbourne, VIC 3122, Australia (e-mails: xge@swin.edu.au; qhan@swin.edu.au).

L. Vlacic is with the School of Engineering and Built Environment, Griffith University, Gold Coast, QLD 4222, Australia (e-mail: 1.vlacic@griffith.edu.au).
(AFS), $x$-by-wire $(\mathrm{XbW})$, and electric power steering (EPS) systems can effectively enhance passenger's safety and driving comfort in AGVs [4]-[7].

The real-time values of yaw rate and sideslip angle are of significance in active vehicle safety technologies because they rely on this information for vehicle lateral risk assessment or adopt it as an input for a vehicle electric control unit. While the vehicle yaw rate can be directly measured by affordable gyroscopes, measurement of the vehicle sideslip angle is only possible through costly sensors such as two-antenna global positioning system (GPS) and optical sensors, which may not be economically viable in mass produced AGVs [8]. An alternative approach is thus needed to accurately estimate the sideslip angle with some relatively inexpensive on-board sensors already available in the vehicle.

Recently, there has been a surge of interest in the modelbased state estimation of vehicle sideslip angle. Up until now, several techniques have been proposed, which can be categorized into two main groups depending on the external disturbance and measurement noise models: 1) stochastic estimation methods (e.g., Kalman filtering and its variants) [9], [10] and 2) non-stochastic estimation methods (e.g., $H_{\infty}$ filtering and energy-to-peak filtering) [11]-[13]. It is well-acknowledged that the performance of stochastic estimation techniques rely heavily on accurate knowledge of particular statistical properties of disturbance/noise, such as mean and covariance of noise terms. However, when it comes to vehicular systems, the real driving conditions and vehicle environments can be complex and hence, the statistical properties of external disturbance and measurement noise are difficult to be accurately estimated. For instance, as an external disturbance, the wind force acting on a vehicle is an irregular random sequence which is influenced by many factors including the windward side of the vehicle, the wind reference area, and the location of the center of pressure in the vehicle body [14]. Therefore, it is quite difficult to exactly know the statistical characteristics of the wind force disturbance in various driving conditions. For the measurement noise, consider a sensor measuring the vehicle yaw rate. At different driving conditions, the variance and standard deviation of the noise in a yaw rate sensor cannot be considered as constant since the measurement noise is not only caused by the physical or electronic characteristic of the sensor, but also by the vibration of vehicle chassis [15]. However, the yaw rate sensor noise can be deterministically bounded since the friction coefficient of the road does not 
always let the vehicle's tyres provide forces to support a high yaw rate [16]. In contrast to stochastic estimation techniques, the majority of non-stochastic estimation methods, particularly $H_{\infty}$ filtering, are based on an assumption of energy bounded disturbance/noise so as to find the worst-case solution to the vehicle state estimation problem [12].

Note that most of the existing estimation methods aim to determine a single-vector with regard to the vehicle state such that the estimation error asymptotically converges to zero and thus, a point-wise estimation of the vehicle state is calculated. Such an estimation approach requires relatively higher sensor precision to steer the vehicle state estimation towards its true state. Besides, no hard bound on disturbance/noise signals means that there is no guarantee of some reliable confidence region enclosing entire distribution of the vehicle state, particularly when there exist external disturbance and measurement noise. This motivates the development of a surrogate technique called ellipsoidal set-membership estimation [17]. This method is aimed at computing a set bounded by an ellipsoid, which includes all possible state estimations while always enclosing the system's true state based on the assumption that disturbance/noise signals are unknown-but-bounded (UBB) [18]. Under this assumption, the only stipulation is to acquire the knowledge of a hard bound on the disturbance/noise signal and hence, the need for gaining a priori knowledge of the rigorous statistical characteristics of disturbance/noise is eliminated. Due to the exceptional power of the set-membership estimation technique in dealing with UBB disturbance/noise signals, such a method has been widely studied for various state estimation formulations in recent years [19]-[25].

Owing to the complexity of modern automated control and sensing systems, the unavoidable existence of faults in AGVs' physical components, i.e., actuators and sensors, jeopardizes vehicle stability and may even result in serious accidents. Examples of such failures are the blocking of an active steering actuator, unavoidable biases in a yaw rate sensor measurement due to uneven road or sensor circuit malfunctions, or a variation in its initial calibration factor due to a temperature drift error [26], [27]. The mitigation of faults requires some knowledge of their quantitative models. This can be fulfilled through implementing suitable fault estimation techniques. Over the last two decades, the problem of fault estimation has received considerable attention from different perspectives [23], [28]-[30]. In the context of vehicular systems, for example, an unknown-input proportional-multi-integral estimator is developed in [31] to estimate the vehicle state and the vehicle's actuator and sensor faults in the existence of some disturbances. By treating the fault and disturbance as an unknown input, it is shown that their superposition can be estimated on condition that there only exists either a fault on the actuator or on the sensor. In [32], a proportional-derivative descriptor estimator is presented for a general discrete-time system to obtain the simultaneous estimates of the system state, actuator fault, sensor fault, and measurement noise. A vehicle dynamic system, which is equipped with the yaw rate and accelerometer sensors, is employed to demonstrate the effectiveness of their proposed method. Recently, a zonotopicbased set-membership fault estimation technique is developed in [23] for a discrete-time linear parameter-varying (LPV) system, where an augmented descriptor system model is presented and the zonotopic estimation interval size is recursively minimized in an effort to improve the estimation performance.

Stability performance of an AGV hinges on reliability and accuracy of sensor and/or estimator outputs and hence, sensors/estimators must guarantee the correctness and availability of the AGV's state. However, in practice, the traditional singlesensor-based estimation technique of vehicle state may be unreliable due to sensor measurement contamination, sensor malfunction, or sensor aging. Therefore, it is of significance to construct a distributed estimation technique to estimate the vehicle state over a multi-sensor network. Such a network configuration is composed of a number of sensor nodes densely deployed in the vehicle. These nodes are linked together through a wired or wireless communication network. Therefore, the distributed estimation over a multi-sensor network has the key feature of utilizing the information collected from several sensors according to a prescribed interaction topology. (see [33]-[37] and the recent survey [38].) In this sense, the estimation accuracy and/or reliability may be improved through information sharing and exchanging among neighboring nodes. To the best of the authors' knowledge, the problem of distributed set-membership joint estimation of vehicle state and sensor fault for AGVs has not been addressed, which motivates the current study.

In this paper, we develop a distributed set-membership estimation technique to jointly estimate an AGV's lateral dynamical state, i.e., the yaw rate and sideslip angle, and sensor faults in the simultaneous presence of both UBB external disturbance and measurement noises. The main contributions are summarized as follows.

1) A joint distributed state and fault estimation configuration is established for an AGV subject to UBB external disturbance and measurement noises. To facilitate the process of sensor fault estimation, an augmented state including the vehicle yaw rate, sideslip angle, and sensor fault signal is formed to construct a descriptor model of the vehicle lateral dynamics in which longitudinal velocity is considered as a time-varying parameter.

2) A distributed set-membership estimation approach is developed so as to improve the reliability and resilience of the state estimates in the presence of sensor saturations and incomplete measurements. It is noteworthy that the proposed approach is capable of estimating each sensor's fault, which may have different quantitative characteristics in practice.

3) A recursive and tractable convex estimation algorithm is derived for jointly estimating vehicle system state and sensor faults in real time. The algorithm aims to find a group of state estimation ellipsoids based on the incomplete and saturated measurement outputs received from individual sensors such that the vehicle's true state and each sensor fault always reside in bounding ellipsoidal sets regardless of the UBB disturbance and noise. The proposed algorithm can also recursively determine the gain matrix sequences for each distributed estimator and shape matrix sequences for optimized ellipsoids.

The remainder of the paper is outlined as follows. Section II 
provides some preliminaries. Section III elaborates the vehicle lateral dynamics, the multi-sensor network configuration and the descriptor system model. Section IV presents the distributed set-membership estimators and also formulates the main problem. Section $\mathrm{V}$ derives the main results in terms of a distributed estimator design criterion and a recursive convex optimization algorithm. Section VI demonstrates the simulation results. Section VII draws a conclusion.

\section{PRELIMINARIES}

\section{A. Notation}

$\mathbb{R}^{n}$ denotes the $n$-dimensional Euclidean space. $\|\cdot\|$ stands for the Euclidean norm. $\operatorname{col}_{n}\left\{x_{i}\right\}$ represents the column vector $\left[x_{1}^{T}, x_{2}^{T}, \ldots, x_{n}^{T}\right]^{T} \cdot \operatorname{diag}_{n}\left\{A_{i}\right\}$ describes the block diagonal matrix $\operatorname{diag}\left\{A_{1}, A_{2}, \ldots, A_{n}\right\} . \mathbb{N}$ represents the set of nonnegative integers. Other notations used are quite standard.

\section{B. Sensor Interaction Topology}

Let the sensor network have $N$ sensing nodes which are densely deployed in the vehicle according to a specific interaction topology represented by a weighted directed graph $\mathcal{G}=(\mathcal{V}, \mathcal{E}, \mathcal{A})$, where $\mathcal{V}=\{1,2, \ldots, N\}$ denotes an index set of nodes, $\mathcal{E} \subseteq \mathcal{V} \times \mathcal{V}$ is an edge set of paired nodes, and $\mathcal{A}=\left[a_{i j}\right] \in \mathbb{R}^{\bar{N} \times N}$ is a weighted adjacency matrix whose elements are positive, i.e., $a_{i j}>0$ iff $(i, j) \in \mathcal{E}$, and $a_{i j}=0$ otherwise. $\mathcal{N}_{i}=\{j \in \mathcal{V}:(i, j) \in \mathcal{E}\}$ denotes the set of neighbors of node $i \in \mathcal{V}$ plus the node itself.

\section{Ellipsoid}

A bounded $n$-dimensional ellipsoid enclosing a real vector $\zeta \in \mathbb{R}^{n}$ is denoted as $\mathscr{E}(c, P, \varrho) \triangleq\left\{\zeta:(\zeta-c)^{T} P^{-1}(\zeta-c) \leq\right.$ $\varrho\}$, where $c \in \mathbb{R}^{n}$ is the center, $P \in \mathbb{R}^{n \times n}$ satisfying $P=P^{T}>0$ is the shape matrix, and $\varrho>0$ is the radius of the ellipsoid, which acts as a scaling parameter of the ellipsoid. The sum of squared semiaxes lengths of the ellipsoid is equivalent to the trace of its shape matrix, i.e., $\operatorname{trace}(P)$. Besides, the size of the ellipsoid is a function of the shape matrix $P$ [39]. Hence, matrix $P$ can be time-varying so as to allow the ellipsoidal bounds to be adjusted over time.

Remark 1: An $n$-dimensional ellipsoid $\mathscr{E}(c, P, \varrho)$ can be viewed as the image of an affine transformation of an ndimensional Euclidean unit ball and has yet another representation as $\mathscr{E} \triangleq\left\{\zeta: \zeta=c+\varrho^{\frac{1}{2}} Q z,\|z\| \leq 1\right\}$, where $Q \in \mathbb{R}^{n \times m}$ with $\operatorname{rank}(Q)=m \leq n$ and satisfying $P=Q Q^{T}>0$ is a lower triangular matrix with positive diagonal elements.

\section{A Multi-Sensor Distributed Estimation CONFIGURATION}

\section{A. Vehicle Lateral Dynamics}

A two-degree-freedom model [16] is adopted to describe the vehicle lateral dynamics, whose schematic is shown in Fig. 1 and vehicle's parameters and their nominal values are described in Table I. Considering small sideslip angle $\beta$ and front wheel steering angle $\delta_{f}$, the lateral and yaw motions can be obtained as

$$
m V_{x}(\dot{\beta}+\dot{\psi})=F_{y f}+F_{y r}, \quad J_{z} \ddot{\psi}=F_{y f} l_{f}-F_{y r} l_{r}
$$

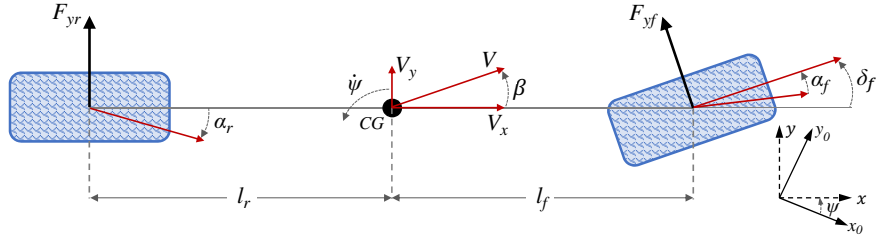

Fig. 1. Schematic of a two-degree-freedom lateral dynamics vehicle model

TABLE I

VEHICLE'S PARAMETER [40]

\begin{tabular}{l|c|l}
\hline Parameter & Symbol & Value-Unit \\
\hline Vehicle's center of gravity & $\mathrm{CG}$ & - \\
Mass of vehicle & $m$ & $1832 \mathrm{~kg}$ \\
Vehicle's yaw moment of inertia & $J_{z}$ & $2488 \mathrm{~kg} \mathrm{~m}^{2}$ \\
Distance between CG and front axle & $l_{f}$ & $1.18 \mathrm{~m}$ \\
Distance between CG and rear axle & $l_{r}$ & $1.77 \mathrm{~m}$ \\
Front/Rear cornering stiffness & $C_{f} / C_{r}$ & $96.240 / 107.180 \mathrm{kN} / \mathrm{rad}$ \\
Front/Rear lateral tire force & $F_{y f} / F_{y r}$ & $\mathrm{~N}$ \\
Front/Rear tire slip angle & $\alpha_{f} / \alpha_{r}$ & $\mathrm{rad}$ \\
\hline
\end{tabular}

where $\psi$ and $\dot{\psi}$ are the vehicle yaw angle and yaw rate, respectively.

With the small front and rear tire slip angles, the total front and rear lateral forces are given by

$$
F_{y f}=-c_{f} \alpha_{f}, \quad F_{y r}=-c_{r} \alpha_{r}
$$

where the tire slip angles can be expressed as

$$
\alpha_{f}=\beta+l_{f} \frac{\dot{\psi}}{V_{x}}-\delta_{f}, \quad \alpha_{r}=\beta-l_{r} \frac{\dot{\psi}}{V_{x}} .
$$

Substituting (2) and (3) into (1) yields the continuous-time state-space model of the vehicle lateral dynamics as

$$
\dot{x}(t)=A^{\prime} x(t)+B^{\prime} u(t)
$$

where the state vector $x(t)=\left[\beta(t)^{T}, \dot{\psi}(t)^{T}\right]^{T}$, the control input $u(t)=\delta_{f}(t)$ and

$$
A^{\prime}=\left[\begin{array}{cc}
-\frac{c_{r}+c_{f}}{m V_{x}} & -1+\frac{l_{r} c_{r}-l_{f} c_{f}}{m V_{x}^{2}} \\
\frac{l_{r} c_{r}-l_{f} c_{f}}{J_{z}} & -\frac{l_{r}^{2} c_{r}+l_{f}^{2} c_{f}}{J_{z} V_{x}}
\end{array}\right], B^{\prime}=\left[\begin{array}{c}
\frac{c_{f}}{m V_{x}} \\
\frac{c_{f} l_{f}}{J_{z}}
\end{array}\right] .
$$

To capture the influence of external disturbances acting on the vehicle, such as wind force, road crown (sloping to one side of the road), and superelevation (banking turn), the external disturbance $w(t)$ is incorporated in model (5) as

$$
\dot{x}(t)=A^{\prime} x(t)+B^{\prime} u(t)+D^{\prime} w(t) .
$$

In this paper, we consider the UBB side wind force as an external disturbance $w(t)$ which may influence the vehicle lateral dynamics. As discussed in [31], the corresponding external disturbance matrix can be represented as $D^{\prime}=$ $\left[f_{w} / m V_{x}, l_{w} f_{w} / J_{z}\right]^{T}$ where $l_{w}$ is the distance of wind force action and $f_{w}$ is the wind force and can be obtained by $f_{w}=V_{w}^{2} A_{s} \rho_{w}$ with $V_{w}, A_{s}$, and $\rho_{w}$ denoting the wind velocity perpendicular to the side surface of the vehicle, the side surface area of the vehicle, and the air density, respectively. 
Note that, in practice, the vehicle longitudinal velocity $V_{x}$ is not always constant which results in time-varying system matrices in (5) as $A^{\prime}(t), B^{\prime}(t)$, and $D^{\prime}(t)$. Hence, model (5) is a nonlinear system with respect to $1 / V_{x}(t)$ and $1 / V_{x}^{2}(t)$. We next employ a Takagi-Sugeno (T-S) fuzzy modeling approach to deal with the nonlinear terms caused by the time-varying longitudinal velocity. To do this, the longitudinal velocity is assumed to be within the range $\left[\underline{V}_{x}, \bar{V}_{x}\right]$, where $\underline{V}_{x}$ and $\bar{V}_{x}$ are known lower bound and upper bound, respectively. In this case, one can obtain that

$\vartheta_{1}=\max \left(1 / V_{x}(t)\right)=1 / \underline{V}_{x}, \quad \vartheta_{2}=\min \left(1 / V_{x}(t)\right)=1 / \bar{V}_{x}$,

$\vartheta_{3}=\max \left(1 / V_{x}^{2}(t)\right)=1 / \underline{V}_{x}^{2}, \quad \vartheta_{4}=\min \left(1 / V_{x}^{2}(t)\right)=1 / \bar{V}_{x}^{2}$.

In light of the sector nonlinear method [41], the nonlinear terms $1 / V_{x}(t)$ and $1 / V_{x}^{2}(t)$ in the system matrices of (5) can be expressed as

$$
\begin{aligned}
& \frac{1}{V_{x}(t)}=\mathcal{M}_{1}\left(\rho_{1}(t)\right) \vartheta_{1}+\mathcal{M}_{2}\left(\rho_{1}(t)\right) \vartheta_{2}, \\
& \frac{1}{V_{x}^{2}(t)}=\mathcal{Q}_{1}\left(\rho_{2}(t)\right) \vartheta_{3}+\mathcal{Q}_{2}\left(\rho_{2}(t)\right) \vartheta_{4}
\end{aligned}
$$

where $\rho_{1}(t)=1 / V_{x}(t)$ and $\rho_{2}(t)=1 / V_{x}^{2}(t)$ represent the premise variables and $\mathcal{M}_{1}\left(\rho_{1}(t)\right), \mathcal{M}_{2}\left(\rho_{1}(t)\right), \mathcal{Q}_{1}\left(\rho_{2}(t)\right)$, and $\mathcal{Q}_{2}\left(\rho_{2}(t)\right)$ denote the membership functions with $\mathcal{M}_{1}\left(\rho_{1}(t)\right)$ and $\mathcal{Q}_{1}\left(\rho_{2}(t)\right)$ stand for "High" longitudinal velocity, and $\mathcal{M}_{2}\left(\rho_{1}(t)\right)$ and $\mathcal{Q}_{2}\left(\rho_{2}(t)\right)$ stand for "Low" longitudinal velocity, respectively. More specifically, the membership functions satisfy

$$
\begin{array}{ll}
\mathcal{M}_{1}\left(\rho_{1}(t)\right)=\frac{\rho_{1}(t)-\vartheta_{2}}{\vartheta_{1}-\vartheta_{2}}, & \mathcal{M}_{2}\left(\rho_{1}(t)\right)=\frac{\vartheta_{1}-\rho_{1}(t)}{\vartheta_{1}-\vartheta_{2}}, \\
\mathcal{Q}_{1}\left(\rho_{2}(t)\right)=\frac{\rho_{2}(t)-\vartheta_{4}}{\vartheta_{3}-\vartheta_{4}}, & \mathcal{Q}_{2}\left(\rho_{2}(t)\right)=\frac{\vartheta_{3}-\rho_{2}(t)}{\vartheta_{3}-\vartheta_{4}}
\end{array}
$$

Then, T-S fuzzy expression of the vehicle lateral dynamics (5) can be given by the following rules

Model Rule 1: IF $\rho_{1}(t)$ is "High" and $\rho_{2}(t)$ is "High" THEN $\dot{x}(t)=A_{1}^{\prime} x(t)+B_{1}^{\prime} u(t)+D_{w, 1}^{\prime} w(t)$

Model Rule 2: IF $\rho_{1}(t)$ is "High" and $\rho_{2}(t)$ is "Low" THEN $\dot{x}(t)=A_{2}^{\prime} x(t)+B_{2}^{\prime} u(t)+D_{w, 2}^{\prime} w(t)$

Model Rule 3: IF $\rho_{1}(t)$ is "Low" and $\rho_{2}(t)$ is "High" THEN $\dot{x}(t)=A_{3}^{\prime} x(t)+B_{3}^{\prime} u(t)+D_{w, 3}^{\prime} w(t)$

Model Rule 4: IF $\rho_{1}(t)$ is "Low" and $\rho_{2}(t)$ is "Low" THEN $\dot{x}(t)=A_{4}^{\prime} x(t)+B_{4}^{\prime} u(t)+D_{w, 4}^{\prime} w(t)$

where the matrices $\left(A_{\ell}^{\prime}, B_{\ell}^{\prime}, D_{w, \ell}^{\prime}\right), \ell=1,2,3,4$, are obtained by replacing the term $1 / V_{x}(t)$ with $\vartheta_{1}$ and $\vartheta_{2}$, and the term $1 / V_{x}(t)^{2}$ with $\vartheta_{3}$ and $\vartheta_{4}$ accordingly in the system matrices $\left(A^{\prime}(t), B^{\prime}(t), D^{\prime}(t)\right)$ in (5).

The overall fuzzy model of the vehicle lateral dynamics is achieved by the fuzzy blending approach as

$$
\dot{x}(t)=\sum_{\ell=1}^{4} \alpha_{\ell}(\rho(t))\left\{A_{\ell}^{\prime} x(t)+B_{\ell}^{\prime} u(t)+D_{w, \ell}^{\prime} w(t)\right\},
$$

where the weighting functions $\alpha_{\ell}(\rho(t)), \ell=1,2,3,4$ satisfy

$$
\begin{aligned}
& \alpha_{1}(\rho(t))=\mathcal{M}_{1}\left(\rho_{1}(t)\right) \cdot \mathcal{Q}_{1}\left(\rho_{2}(t)\right), \\
& \alpha_{2}(\rho(t))=\mathcal{M}_{1}\left(\rho_{1}(t)\right) \cdot \mathcal{Q}_{2}\left(\rho_{2}(t)\right), \\
& \alpha_{3}(\rho(t))=\mathcal{M}_{2}\left(\rho_{1}(t)\right) \cdot \mathcal{Q}_{1}\left(\rho_{2}(t)\right), \\
& \alpha_{4}(\rho(t))=\mathcal{M}_{2}\left(\rho_{1}(t)\right) \cdot \mathcal{Q}_{2}\left(\rho_{2}(t)\right),
\end{aligned}
$$

One can verify that $\alpha_{\ell}(\rho(t)) \geq 0$ and $\sum_{\ell=1}^{4} \alpha_{\ell}(\rho(t))=1$.

To facilitate the subsequent distributed set-membership estimator design, we further discretize the T-S fuzzy model of the vehicle lateral dynamics with a constant sampling period $\tau_{s}$. To do so, the system matrices in (6) at the sampling period $\tau_{s}$ can be obtained by

$A_{\ell}=e^{A_{\ell}^{\prime} \tau_{s}}, B_{\ell}=\int_{0}^{\tau_{s}} e^{A_{\ell}^{\prime} s} d s B_{\ell}^{\prime}, D_{w, \ell}=\int_{0}^{\tau_{s}} e^{A_{\ell}^{\prime} s} d s D_{w, \ell}^{\prime}$ and $u_{k}=u\left(t_{k}\right)$ is sampled at the sampling instants $t_{k}=$ $k \tau_{s}$. The UBB side wind force disturbance is assumed to be confined to the following ellipsoid:

$$
\mathscr{W}_{k} \triangleq\left\{w_{k}: w_{k}^{T} W_{k}^{-1} w_{k} \leq 1\right\} .
$$

Hence, the discretized T-S fuzzy model of the vehicle lateral dynamics can be expressed as

$$
x_{k+1}=\sum_{\ell=1}^{4} \alpha_{\ell}\left(\rho_{k}\right)\left\{A_{\ell} x_{k}+B_{\ell} u_{k}+D_{w, \ell} w_{k}\right\} .
$$

\section{B. A Multi-Sensor Network Configuration}

For each $i \in \mathcal{V}$, the measurement output $y_{i, k} \in \mathbb{R}^{n_{y}}$ of sensor $i$ in the presence of the sensor fault is given by

$$
y_{i, k}=C_{i} x_{k}+D_{s, i} f_{i, k}^{s}+D_{v, i} v_{i, k}
$$

where $f_{i, k}^{s} \in \mathbb{R}^{n_{f}}$ denotes the fault in sensor $i$; $D_{s, i}$ represents the sensor $i$ fault matrix with an appropriate dimension; and the measurement noise $v_{i, k} \in \mathbb{R}^{n_{v}}$ is UBB and enclosed by the following ellipsoid:

$$
\mathscr{V}_{k}^{i} \triangleq\left\{v_{i, k}: v_{i, k}^{T} R_{i, k}^{-1} v_{i, k} \leq 1\right\} .
$$

Remark 2: As discussed in [8], the yaw rate measurement is an appropriate choice for the sideslip angle estimation since the commercialized gyroscope can measure the yaw rate with no extra cost. Hence, the related element to the sideslip angle in matrix $C_{i}$ can be set as zero, which indicates that sensor $i$ only measures the yaw rate and thus $n_{y}=1$. In practice, the yaw rate sensors can be typically located either in the vehicle's electric control unit, under the dashboard near the fuse box, or below the driver's and/or the front passenger's seats, mounted on the level floorboard in order to access the vehicle's center of gravity [42].

\section{A Descriptor System Model}

To facilitate the sensor fault estimation process, the fault vector $f_{i, k}^{s}$ can be regarded as an auxiliary state and hence, an augmented state vector can be constructed as $\bar{x}_{i, k}=$ 
$\left[x_{k}^{T}, f_{i, k}^{s}\right]^{T}$. Then, the vehicle lateral dynamics described by (8) can be represented by a descriptor system as

$$
E \bar{x}_{i, k+1}=\sum_{\ell=1}^{4} \alpha_{\ell}\left(\rho_{k}\right)\left(\bar{A}_{\ell} \bar{x}_{i, k}+\bar{B}_{\ell} u_{k}+\bar{D}_{w, \ell} w_{k}\right)
$$

where

$$
E=\left[\begin{array}{ll}
I & 0 \\
0 & 0
\end{array}\right], \bar{A}_{\ell}=\left[\begin{array}{cc}
A_{\ell} & 0 \\
0 & 0
\end{array}\right], \bar{B}_{\ell}=\left[\begin{array}{c}
B_{\ell} \\
0
\end{array}\right], \bar{D}_{w, \ell}=\left[\begin{array}{c}
D_{w, \ell} \\
0
\end{array}\right] .
$$

It is accepted that the measurement output of each sensor may be incomplete due to signal shading and channel fading [43], and/or network-induced data packet losses [44]. In addition, sensors may suffer from a common phenomenon known as "sensor saturation", when measured signals lie outside the sensor's dynamic range. Sensor saturation affects the estimation performance of the designed estimator, and may also cause instability of the system. Therefore, the incomplete measurement of sensor $i$ in the presence of sensor saturation can be expressed as

$$
\tilde{y}_{i, k}=\gamma_{i, k} \sigma\left(\bar{C}_{i} \bar{x}_{i, k}\right)+D_{v, i} v_{i, k}
$$

where $\bar{C}_{i}=\left[\begin{array}{ll}C_{i} & D_{s, i}\end{array}\right]$, and $\gamma_{i, k} \in(0,1]$ represents the availability status of the measurement output of sensor $i$. If $\gamma_{i, k}=1$, the measurement output of sensor $i$ is completely available at time $k$. When $0<\gamma_{i, k}<1$, the sensor $i$ 's measurement output is partially available at time $k$. Note that incorporating the measurement status parameter $\gamma_{i, k}$ allows the system designer to characterize different levels of incomplete measurements and evaluate their impact on the performance of the designed estimators.

The saturation function $\sigma(\cdot): \mathbb{R}^{n_{y}} \mapsto \mathbb{R}^{n_{y}}$ for sensor $i$ in (12) is defined as

$$
\sigma\left(r_{i}\right)=\left[\sigma_{1}\left(r_{1 i}\right), \ldots, \sigma_{n_{y}}\left(r_{n_{y} i}\right)\right]^{T} .
$$

Since $n_{y}=1$, the saturation function $\sigma\left(r_{i}\right)=\sigma_{1}\left(r_{1 i}\right)$ and can be expressed as $\sigma\left(r_{i}\right)=\operatorname{sign}\left(r_{i}\right) \min \left\{r_{i, \max },\left|r_{i}\right|\right\}$. It is assumed that there exist scalar sequences $h_{1 i}$ and $h_{2 i}$ satisfying $0<h_{1 i}<1 \leq h_{2 i}$ such that the saturation function $\sigma\left(\bar{C}_{i} \bar{x}_{i, k}\right)$ in (12) can be written as

$$
\sigma\left(\bar{C}_{i} \bar{x}_{i, k}\right)=h_{1 i} \bar{C}_{i} \bar{x}_{i, k}+\varphi\left(\bar{C}_{i} \bar{x}_{i, k}\right)
$$

where $\varphi\left(\bar{C}_{i} \bar{x}_{i, k}\right)$ is a nonlinear vector-valued function which satisfies a sector condition $\left[0, h_{i}\right]$ with $h_{i}=h_{2 i}-h_{1 i}$, i.e., $\varphi\left(\bar{C}_{i} \bar{x}_{i, k}\right)$ satisfies the following inequality:

$$
\varphi^{T}\left(\bar{C}_{i} \bar{x}_{i, k}\right)\left(\varphi\left(\bar{C}_{i} \bar{x}_{i, k}\right)-h_{i} \bar{C}_{i} \bar{x}_{i, k}\right) \leq 0 .
$$

For brevity, denote $\sigma\left(\bar{C}_{i} \bar{x}_{i, k}\right)=\sigma_{i, k}$ and $\varphi\left(\bar{C}_{i} \bar{x}_{i, k}\right)=\varphi_{i, k}$.

\section{Distributed Set-membership Joint State and FAULT ESTIMATION PROBLEM}

It is our interest to develop a distributed set-membership estimation approach which includes a group of estimators computing some ellipsoidal sets of the augmented state estimation including vehicle state estimation and sensor fault estimation based on local measurement of neighboring yaw rate sensors. Each sensing node $i \in \mathcal{V}$ consists of a processing unit, a buffer, and an estimator as depicted in Fig. 2. Suppose

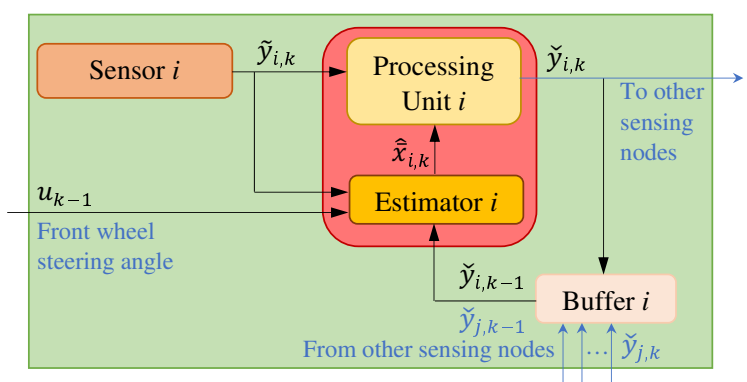

Fig. 2. Architecture of the $i$ th sensing node in an AGV equipped with a multi-sensor network

that the desired fuzzy estimator $i$ attached to sensor $i$ has the following form

$$
\begin{array}{r}
\hat{\bar{x}}_{i, k+1}=\sum_{\ell=1}^{4} \alpha_{\ell}\left(\rho_{k}\right)\left(T_{i, k} \bar{A}_{\ell} \hat{\bar{x}}_{i, k}+T_{i, k} \bar{B}_{\ell} u_{k}+G_{i, k} \tilde{y}_{i, k+1}\right. \\
\left.+L_{i \ell} \sum_{j \in \mathcal{N}_{i}} a_{i j} \check{y}_{j, k}\right)
\end{array}
$$$$
\check{y}_{i, k}=\tilde{y}_{i, k}-\bar{C}_{i} \hat{\bar{x}}_{i, k}
$$

for all $k \in \mathbb{N}$, where $\hat{\bar{x}}_{i, k} \in \mathbb{R}^{\left(n_{x}+n_{f}\right)}$ is the calculated estimate of the augmented state $\bar{x}_{i, k}$ at the $i$ th sensing node; $\check{y}_{i, k}$ is the output of the processing unit $i$; the buffer in Fig. 2 stores data in a last-in-first-out manner and sends the one-step-behind data received from its own sensing node and the other nodes to estimator $i ; T_{i, k}$ and $G_{i, k}$ are the estimator matrix sequences to be designed; $L_{i \ell}$ is the estimator $i$ 's gain matrix sequence to be determined; and $\hat{\bar{x}}_{i, 0}$ is the given initial condition, which satisfies the following assumption.

Assumption 1: The initial augmented state estimate $\hat{\bar{x}}_{i, 0}$ belongs to a given ellipsoid

$$
\mathscr{X}_{0}^{i} \triangleq\left\{\bar{x}_{i, 0}:\left(\bar{x}_{i, 0}-\hat{\bar{x}}_{i, 0}\right)^{T} P_{i, 0}^{-1}\left(\bar{x}_{i, 0}-\hat{\bar{x}}_{i, 0}\right) \leq \varrho_{i}\right\}
$$

where $P_{i, 0}$ is a known shape matrix.

Next, we present the following lemma, which will be employed to find the solutions for matrices $T_{i, k}$ and $G_{i, k}$.

Lemma 1: [45] For given matrices $\mathcal{B}$ and $\mathcal{Z}$, there exists a matrix $\mathcal{Y}$ such that $\mathcal{Y} \mathcal{B}=\mathcal{Z}$ if and only if

$$
\operatorname{rank}\left[\begin{array}{l}
\mathcal{B} \\
\mathcal{Z}
\end{array}\right]=\operatorname{rank}(\mathcal{Z})
$$

A general solution to $\mathcal{Y} \mathcal{B}=\mathcal{Z}$ is given by

$$
\mathcal{Y}=\mathcal{Z} \mathcal{B}^{\dagger}+\mathcal{S}\left(I-\mathcal{B B}^{\dagger}\right)
$$

where $\mathcal{B}^{\dagger}$ is the pseudo-inverse of $\mathcal{B}$ and $\mathcal{S}$ is an arbitrary matrix with an appropriate dimension.

Remark 3: Since the descriptor system described by (8) and (9) satisfies that

$$
\operatorname{rank}\left[\begin{array}{c}
E \\
\gamma_{i, k+1} h_{1 i} \bar{C}_{i}
\end{array}\right]=n_{x}+n_{f}
$$

from Lemma 1, there exists a matrix $\left[\begin{array}{ll}T_{i, k} & G_{i, k}\end{array}\right]$ such that

$$
\left[\begin{array}{ll}
T_{i, k} & G_{i, k}
\end{array}\right]\left[\begin{array}{c}
E \\
\gamma_{i, k+1} H_{1 i} \bar{C}_{i}
\end{array}\right]=I_{n_{x}+n_{f}}
$$


which is equivalent to

$$
T_{i, k} E+G_{i, k} \gamma_{i, k+1} h_{1 i} \bar{C}_{i}=I_{n_{x}+n_{f}} .
$$

By using Lemma 1 , matrices $T_{i, k} \in \mathbb{R}^{\left(n_{x}+n_{f}\right) \times\left(n_{x}+n_{f}\right)}$ and $G_{i, k} \in \mathbb{R}^{\left(n_{x}+n_{f}\right) \times n_{y}}$ can be determined by

$$
\begin{aligned}
& T_{i, k}=\Theta_{i, k}^{\dagger} \epsilon_{1}+S\left(I_{n_{x}+n_{f}+n_{y}}-\Theta_{i, k} \Theta_{i, k}^{\dagger}\right) \epsilon_{1} \\
& G_{i, k}=\Theta_{i, k}^{\dagger} \epsilon_{2}+S\left(I_{n_{x}+n_{f}+n_{y}}-\Theta_{i, k} \Theta_{i, k}^{\dagger}\right) \epsilon_{2}
\end{aligned}
$$

where $S \in \mathbb{R}^{\left(n_{x}+n_{f}\right) \times\left(n_{x}+n_{f}+n_{y}\right)}$ is an arbitrary matrix which provides more degrees of freedom, matrices $\Theta_{i, k} \in$ $\mathbb{R}^{\left(n_{x}+n_{f}+n_{y}\right) \times\left(n_{x}+n_{f}\right)}, \epsilon_{1} \in \mathbb{R}^{\left(n_{x}+n_{f}+n_{y}\right) \times\left(n_{x}+n_{f}\right)}$, and $\epsilon_{2} \in$ $\mathbb{R}^{\left(n_{x}+n_{f}+n_{y}\right) \times n_{y}}$ are given by

$$
\Theta_{i, k}=\left[\begin{array}{c}
E \\
\gamma_{i, k+1} h_{1 i} \bar{C}_{i}
\end{array}\right], \epsilon_{1}=\left[\begin{array}{c}
I_{n_{x}+n_{f}} \\
0
\end{array}\right], \epsilon_{2}=\left[\begin{array}{c}
0 \\
I_{n_{y}}
\end{array}\right] .
$$

To proceed with the concerned distributed set-membership joint state and sensor fault estimation problem, we next introduce the following definition.

Definition 1: The vehicle described by (8) and (9) subject to UBB external disturbance $w_{k} \in \mathscr{W}_{k}$ and measurement noise $v_{i, k} \in \mathscr{V}_{k}^{i}$ achieves the distributed set-membership state estimation on each node $i, \forall i \in \mathcal{V}$, if there exists a gain sequence $L_{i \ell}$ such that the vehicle's one-step ahead state $\bar{x}_{i, k+1}$ is enclosed in a state estimation ellipsoid computed by node $i$, that is $\mathscr{X}_{k+1}^{i}$, which always contains the true augmented state of the vehicle, where

$$
\mathscr{X}_{k+1}^{i} \triangleq\left\{\bar{x}_{i, k+1}: e_{i, k+1}^{T} P_{i, k+1}^{-1} e_{i, k+1} \leq \varrho_{i}\right\}
$$

for all $k \in \mathbb{N}$, where $e_{i, k+1}=\bar{x}_{i, k+1}-\hat{\bar{x}}_{i, k+1}$ represents an estimation error vector on vehicle's sensing node $i$ and $P_{i, k+1}=P_{i, k+1}^{T}>0$ is a time-varying shape matrix.

The proposed distributed set-membership joint state and fault estimation problem can now be stated as: For a given scalar $\varrho_{i}>0$, UBB disturbance $w_{k} \in \mathscr{W}_{k}$ and measurement noise $v_{i, k} \in \mathscr{V}_{k}^{i}$, the objective is to find a suitable shape matrix sequence $P_{i, k+1}$ and estimator $i$ 's state $\hat{\bar{x}}_{i, k+1}$ such that the specified ellipsoid $\mathscr{X}_{k+1}^{i}$ always confines the vehicle's true augmented state $\bar{x}_{i, k+1}$.

\section{MAin Results}

In this section, a theorem will be first established to provide a sufficient condition on the existence of the desired distributed estimators formulated in (16) such that (21) holds. Then, an algorithm will be presented so as to numerically solve the concerned distributed set-membership joint state and sensor fault estimation problem in a recursive manner.

To simplify subsequent analysis, we define the following notations:

$$
\begin{aligned}
& \bar{x}_{k}=\operatorname{col}_{N}\left\{\bar{x}_{i, k}\right\}, \hat{\bar{x}}_{k}=\operatorname{col}_{N}\left\{\hat{\bar{x}}_{i, k}\right\}, \tilde{e}_{k}=\operatorname{col}_{N}\left\{e_{i, k}\right\} \\
& \tilde{w}_{k}=\operatorname{col}_{N}\left\{w_{k}\right\}, \tilde{v}_{k}=\operatorname{col}_{N}\left\{v_{i, k}\right\}, \tilde{z}=\operatorname{col}_{N}\left\{z_{i}\right\} \\
& U_{k}=\operatorname{col}_{N}\left\{u_{k}\right\}, Y_{k}=\operatorname{col}_{N}\left\{\tilde{y}_{i, k}\right\}, \tilde{\varrho}=\operatorname{diag}_{N}\left\{\varrho_{i}^{\frac{1}{2}}\right\} \\
& \tilde{W}_{k}=\operatorname{diag}_{N}\left\{W_{k}\right\}, \tilde{R}_{k}=\operatorname{diag}_{N}\left\{R_{i, k}\right\}, L_{\ell}=\operatorname{diag}_{N}\left\{L_{i \ell}\right\} \\
& \tilde{P}_{k}=\operatorname{diag}_{N}\left\{P_{i, k}\right\}, \tilde{M}_{k}=\operatorname{diag}_{N}\left\{M_{i, k}\right\}, \tilde{C}=\operatorname{diag}_{N}\left\{\bar{C}_{i}\right\} \\
& \tilde{A}_{\ell}=\operatorname{diag}_{N}\left\{\bar{A}_{\ell}\right\}, \tilde{B}_{\ell}=\operatorname{diag}_{N}\left\{\bar{B}_{\ell}\right\} \tilde{D}_{w, \ell}=\operatorname{diag}_{N}\left\{\bar{D}_{w, \ell}\right\}
\end{aligned}
$$

$\tilde{D}_{v}=\operatorname{diag}_{N}\left\{D_{v, i}\right\}, \tilde{T}_{k}=\operatorname{diag}_{N}\left\{T_{i, k}\right\}, \tilde{G}_{k}=\operatorname{diag}_{N}\left\{G_{i, k}\right\}$

$H_{1}=\operatorname{diag}_{N}\left\{h_{1 i}\right\}, H=\operatorname{diag}_{N}\left\{h_{i}\right\}, \tilde{\varphi}_{k}=\operatorname{diag}_{N}\left\{\varphi_{i, k}\right\}$

$\Gamma_{k}=\operatorname{diag}_{N}\left\{\gamma_{i, k}\right\}$.

\section{A. Design Criterion}

The following theorem outlines the sufficient condition on the existence of an optimized ellipsoidal estimate set $\mathscr{X}_{k+1}^{i}$ at each sampling time on account of UBB external disturbance, UBB measurement noise, and incomplete and saturated measurement outputs received from individual sensors.

Theorem 1: For the vehicle lateral dynamics expressed by (8) and (9) subject to $w_{k} \in \mathscr{W}_{k}$ and $v_{i, k} \in \mathscr{V}_{k}^{i}$, suppose that at time $k$ the augmented state $\bar{x}_{i, k}$ belongs to its state estimation ellipsoid $\mathscr{X}_{k}^{i}$. Then, the one-step ahead augmented state $\bar{x}_{i, k+1}$ always resides in the state estimation ellipsoid $\mathscr{X}_{k+1}^{i}$, if there exist matrix sequences $P_{i, k+1}, L_{i \ell}$, and scalar sequences $\tau_{m, k}^{\ell}>0, m=1,2, \ldots, 6$, such that the following convex optimization problem (OP) is solvable

$$
\begin{aligned}
& \min _{\tilde{P}_{k+1}, L_{\ell}, \tau_{m, k}^{\ell}} \operatorname{trace}\left(\tilde{P}_{k+1}\right) \\
& \text { subject to }\left[\begin{array}{cc}
-\tilde{P}_{k+1} & \Pi_{\ell, k} \\
* & \Psi_{\ell, k}
\end{array}\right] \leq 0
\end{aligned}
$$

for each T-S fuzzy model rule $\ell=1,2,3,4$ and all $k \in \mathbb{N}$, where

$$
\begin{aligned}
& \Pi_{\ell, k}=\left[L_{\ell} \mathcal{A}\left(I-\Gamma_{k} H_{1}\right) \tilde{C} \hat{\bar{x}}_{k} \quad \tilde{\varrho}\left(\tilde{T}_{k} \tilde{A}_{\ell}-L_{\ell} \mathcal{A} \Gamma_{k} H_{1} \tilde{C}\right) \tilde{M}_{k}\right. \\
& \left.\tilde{T}_{k} \tilde{D}_{w, \ell}-L_{\ell} \mathcal{A} \tilde{D}_{v} \quad-\tilde{G}_{k} \tilde{D}_{v} \quad-L_{\ell} \mathcal{A} \Gamma_{k} \quad-\tilde{G}_{k} \Gamma_{k+1}\right]
\end{aligned}
$$

and $\Psi_{\ell, k}=\left[\Psi_{p, q}^{\ell}(k)\right]_{7 \times 7}$ is a time-varying sparse symmetric block matrix with its nonzero entries given by

$$
\begin{aligned}
& \Psi_{1,1}^{\ell}(k)=-\sum_{i=1}^{N} \varrho_{i}+N \sum_{\lambda=1}^{4} \tau_{\lambda, k}^{\ell} \\
& \Psi_{1,6}^{\ell}(k)=\tau_{5, k}^{\ell} \Phi_{1,6}(k), \Psi_{1,7}^{\ell}(k)=\tau_{6, k}^{\ell} \Upsilon_{1,7}^{\ell}(k) \\
& \Psi_{2,2}^{\ell}(k)=-\tau_{1, k}^{\ell} I, \Psi_{2,6}^{\ell}(k)=\tau_{5, k}^{\ell} \Phi_{2,6}(k) \\
& \Psi_{2,7}^{\ell}(k)=\tau_{6, k}^{\ell} \Upsilon_{2,7}^{\ell}(k), \Psi_{3,3}^{\ell}(k)=-\tau_{2, k}^{\ell} \tilde{W}_{k}^{-1} \\
& \Psi_{3,7}^{\ell}(k)=\tau_{6, k}^{\ell} \Upsilon_{3,7}^{\ell}(k), \Psi_{4,4}^{\ell}(k)=-\tau_{3, k}^{\ell} \tilde{R}_{k}^{-1} \\
& \Psi_{5,5}^{\ell}(k)=-\tau_{4, k}^{\ell} \tilde{R}_{k+1}^{-1}, \Psi_{5,7}^{\ell}(k)=\tau_{6, k}^{\ell} \Upsilon_{5,7}^{\ell}(k) \\
& \Psi_{6,6}^{\ell}(k)=\tau_{5, k}^{\ell} \Phi_{6,6}(k), \Psi_{7,7}^{\ell}(k)=\tau_{6, k}^{\ell} \Upsilon_{7,7}^{\ell}(k)
\end{aligned}
$$

where $\Phi_{k}$ and $\Upsilon_{k}$ are defined in (27).

Proof: At time $k$, suppose that the augmented state $\bar{x}_{i, k}$ belongs to its state estimation ellipsoid $\mathscr{X}_{k}^{i}$ and satisfies $e_{i, k}^{T} P_{i, k}^{-1} e_{i, k} \leq \varrho_{i}$, that is $\left(\bar{x}_{i, k}-\hat{\bar{x}}_{i, k}\right)^{T} P_{i, k}^{-1}\left(\bar{x}_{i, k}-\hat{\bar{x}}_{i, k}\right) \leq \varrho_{i}$. Applying a Cholesky factorization, one has $P_{i, k}=M_{i, k} M_{i, k}^{T}$. Letting $z_{i}=\varrho_{i}^{-1 / 2} M_{i, k}^{-1}\left(\bar{x}_{i, k}-\hat{\bar{x}}_{i, k}\right)$, it is straightforward to verify that

$$
z_{i}^{T} z_{i}=\varrho_{i}^{-1}\left(\bar{x}_{i, k}-\hat{\bar{x}}_{i, k}\right)^{T} P_{i, k}^{-1}\left(\bar{x}_{i, k}-\hat{\bar{x}}_{i, k}\right) \leq 1,
$$

and thus there exists a vector $z_{i}$ satisfying $\left\|z_{i}\right\| \leq 1$ such that

$$
\bar{x}_{i, k}=\hat{\bar{x}}_{i, k}+\varrho_{i}^{\frac{1}{2}} M_{i, k} z_{i} .
$$


From (8), (16), (18), and (24), the one-step ahead state estimation error for each T-S fuzzy model rule $\ell$ can be calculated as

$$
\begin{aligned}
e_{i, k+1}= & T_{i, k} \bar{A}_{\ell}\left(\bar{x}_{i, k}-\hat{\bar{x}}_{i, k}\right)+T_{i, k} \bar{D}_{w, \ell} w_{k} \\
& -G_{i, k} \gamma_{i, k+1} \varphi_{i, k+1}-G_{i, k} D_{v, i} v_{i, k+1} \\
& -L_{i \ell} \sum_{j \in \mathcal{N}_{i}} a_{i j}\left(\tilde{y}_{j, k}-\bar{C}_{j} \hat{\bar{x}}_{j, k}\right) \\
= & T_{i, k} \bar{A}_{\ell} \varrho_{i}^{\frac{1}{2}} M_{i, k} z_{i}+T_{i, k} \bar{D}_{w, \ell} w_{k}-G_{i, k} D_{v, i} v_{i, k+1} \\
& -G_{i, k} \gamma_{i, k+1} \varphi_{i, k+1}-L_{i \ell} \sum_{j \in \mathcal{N}_{i}} a_{i j}\left(\left(\gamma_{j, k} h_{1 j}-I\right)\right. \\
& \bar{C}_{j} \hat{\bar{x}}_{j, k}+\gamma_{j, k} h_{1 j} \bar{C}_{j} \varrho_{j}^{1 / 2} M_{j, k} z_{j}+\gamma_{j, k} \varphi_{j, k} \\
& \left.+D_{v, j} v_{j, k}\right) .
\end{aligned}
$$

Denoting $\eta_{k}=\left[1, \tilde{z}^{T}, \tilde{w}_{k}^{T}, \tilde{v}_{k}^{T}, \tilde{v}_{k+1}^{T}, \tilde{\varphi}_{k}^{T}, \tilde{\varphi}_{k+1}^{T}\right]^{T}$, from (25), it can be shown that $\tilde{e}_{k+1}=\Pi_{\ell, k} \eta_{k}$. Thus, for T-S fuzzy model rule $\ell$, the quadratic one-step ahead estimation error $e_{i, k+1}^{T} P_{i, k+1}^{-1} e_{i, k+1} \leq \varrho_{i}$ in (21) can be rewritten as

$$
\eta_{k}^{T}\left(\Pi_{\ell, k}^{T} \tilde{P}_{k+1}^{-1} \Pi_{\ell, k}+\bar{\Omega}\right) \eta_{k} \leq 0
$$

where $\bar{\Omega}=\operatorname{diag}\left\{-\sum_{i=1}^{N} \varrho_{i}, 0,0,0,0,0,0\right\}$.

From $\left\|z_{i}\right\| \leq 1$, (7), and (10), we have the following constraints that $\eta_{k}^{T} \Xi_{1, k} \eta_{k} \geq 0, \eta_{k}^{T} \Xi_{2, k} \eta_{k} \geq 0$, $\eta_{k}^{T} \Xi_{3, k} \eta_{k} \geq 0$, and $\eta_{k}^{T} \Xi_{4, k} \eta_{k} \geq 0$, where $\Xi_{1, k}=$ $\operatorname{diag}\{N,-I, 0,0,0,0,0\}, \Xi_{2, k}=\operatorname{diag}\left\{N, 0,-\tilde{W}_{k}^{-1}, 0,0,0\right.$, $0\}, \Xi_{3, k}=\operatorname{diag}\left\{N, 0,0,-\tilde{R}_{k}^{-1}, 0,0,0\right\}, \Xi_{4, k}=\operatorname{diag}\{N, 0,0$, $\left.0,-\tilde{R}_{k+1}^{-1}, 0,0\right\}$.

From (15), the conditions on the unknown nonlinear vectorvalued functions, $\varphi_{i, k}$ and $\varphi_{i, k+1}$ can be written as $\eta_{k}^{T} \Phi_{k} \eta_{k} \geq$ 0 and $\eta_{k}^{T} \Upsilon_{k}^{\ell} \eta_{k} \geq 0$ for all $i \in \mathcal{V}$, respectively, where $\Phi_{k}=\left[\Phi_{p_{1}, q_{1}}(k)\right]_{7 \times 7}$ and $\Upsilon_{k}^{\ell}=\left[\Upsilon_{p_{2}, q_{2}}^{\ell}(k)\right]_{7 \times 7}$ are timevarying sparse symmetric block matrices with their nonzero entries given by

$$
\begin{aligned}
& \Phi_{1,6}(k)=\frac{1}{2} \hat{\bar{x}}_{k}^{T} \tilde{C}^{T} H, \Phi_{2,6}(k)=\tilde{M}_{k}^{T} \tilde{\varrho} \tilde{C}^{T} H, \Phi_{6,6}(k)=-I \\
& \Upsilon_{1,7}^{\ell}(k)=\frac{1}{2}\left(\hat{\bar{x}}_{k}^{T} \tilde{A}_{\ell}^{T} \tilde{T}_{k}^{T}+U_{k}^{T} \tilde{B}_{\ell}^{T} \tilde{T}_{k}^{T}+Y_{k+1}^{T} \tilde{G}_{k}^{T}\right) \tilde{C}^{T} H \\
& \Upsilon_{2,7}^{\ell}(k)=\frac{1}{2} \tilde{M}_{k}^{T} \tilde{\varrho} \tilde{A}_{\ell}^{T} \tilde{T}_{k}^{T} \tilde{C}^{T} H, \Upsilon_{3,7}^{\ell}(k)=\frac{1}{2} \tilde{D}_{w, \ell}^{T} \tilde{T}_{k}^{T} \tilde{C}^{T} H \\
& \Upsilon_{5,7}^{\ell}(k)=-\frac{1}{2} \tilde{D}_{v}^{T} \tilde{G}_{k}^{T} \tilde{C}^{T} H, \Upsilon_{7,7}^{\ell}(k)=\left(I+H \tilde{C} \tilde{G}_{k} \Gamma_{k}\right) .
\end{aligned}
$$

By virtue of $\mathcal{S}$-procedure, (26) holds if there exist positive scalar sequences $\tau_{m, k}^{\ell}, m=1,2, \ldots, 6$, such that

$$
\begin{gathered}
\Pi_{\ell, k}^{T} \tilde{P}_{k+1}^{-1} \Pi_{\ell, k}+\bar{\Omega}+\tau_{1, k}^{\ell} \Xi_{1, k}+\tau_{2, k}^{\ell} \Xi_{2, k}+\tau_{3, k}^{\ell} \Xi_{3, k} \\
\quad+\tau_{4, k}^{\ell} \Xi_{4, k}+\tau_{5, k}^{\ell} \Phi_{k}+\tau_{6, k}^{\ell} \Upsilon_{k} \leq 0,
\end{gathered}
$$

which can be written as the inequality in (22) by virtue of the Schur complement [46]. In order to derive some optimized ellipsoids and reduce the conservativeness, OP (22) is introduced to minimize the shape matrix $\tilde{P}_{k+1}$ in the sense of matrix trace. This completes the proof.

Remark 4: In light of Theorem 1, the proposed distributed joint state and sensor fault estimation problem can be cast into the feasibility problem of a set of recursive linear matrix inequality (RLMI) in (22). Through Theorem 1, one can recursively solve out the estimator $i$ 's gain matrix sequence $L_{i \ell}$ for each T-S fuzzy model rule $\ell$ and determine optimized state estimation ellipsoids $\mathscr{X}_{k+1}^{i}$ enclosing the true augmented state of the vehicle despite the existence of UBB external disturbance, UBB measurement noise, incomplete measurement, and sensor saturation. Since the inequality in (22) is linear to $\tilde{P}_{k+1}$, $L_{\ell}$, and $\tau_{m, k}^{\ell}, m=1, \ldots, 6$, one can numerically solve OP (22) by resorting to some existing semidefinite programming via an interior-point algorithm at each time step. As a result, Theorem 1 provides a criterion for designing the desired setmembership joint state and fault estimator introduced in (16) and confidence augmented state estimation ellipsoids $\mathscr{X}_{k+1}^{i}$ presented in (21) for the vehicle's one-step ahead augmented state $\bar{x}_{i, k+1}$.

Remark 5: The interior-point algorithm usually has a polynomial-time complexity $\mathcal{O}\left(\mathcal{R} \mathcal{M}^{3}\right)$, where $\mathcal{R}$ is the total row size of the main LMIs, $\mathcal{M}$ is the total number of scalar decision variables of the main LMI (22). Since $\mathcal{R}$ and $\mathcal{M}$ are dependent on $n_{x}, n_{u}, n_{y}, n_{w}, n_{v}$, and $n_{f}$, the computational complexity of the developed recursive algorithm depends polynomially on the dimensions of each sensing node's parameter variables. Furthermore, the feasibility of Theorem 1 must be checked for each sensing node $i, \forall i \in \mathcal{V}$ and thus, the total computation complexity of the main LMI (22) linearly depends on the number of sensing nodes.

\section{B. Recursive Convex Optimization Algorithm}

Based on (22), we next present a recursive distributed algorithm, i.e., Algorithm 1, that computes the real-time optimized augmented state estimation ellipsoids based on each sensor's incomplete and saturated measurement output. Algorithm 1 generates the gain matrix for estimator $i$ (16) and determines its shape matrix sequences $P_{i, k+1}$.

\section{Simulation Results and Case Studies}

In this section, the developed distributed set-membership estimation approach is applied to investigate the joint state and sensor fault estimation problem of an AGV whose required parameters' values are given in Table. I. A network of six sensing nodes, i.e., $\mathcal{V}=\{1,2, \ldots, 6\}$, are deployed to cooperatively sense the vehicle yaw rate. The interaction topology of these nodes is demonstrated in Fig. 3, where the adjacency element $a_{i j}$ has a binary value.

In simulation, the vehicle longitudinal velocity $V_{x}(t)$ is assumed to be within the range $[80,120] \mathrm{km} / \mathrm{h}$ as depicted in Fig. 4. Furthermore, the controller sends a random front wheel steering angle as $u_{k}=0.0175+(0.0542-0.0175) \operatorname{rand}(1,1)$, which implies that the front wheels are turned from left to right frequently within the range $\left[1^{\circ}, 3^{\circ}\right]$. Given a sampling time of $\tau_{s}=0.1 \mathrm{~s}$, the corresponding discrete-time system matrices in (8) for each T-S fuzzy model rule $\ell$ are obtained as

$$
\begin{aligned}
& A_{1}=\left[\begin{array}{cc}
0.6212 & -0.0586 \\
1.8617 & 0.4792
\end{array}\right], B_{1}=\left[\begin{array}{c}
-0.0317 \\
3.5065
\end{array}\right] \\
& A_{2}=\left[\begin{array}{cc}
0.6257 & -0.0558 \\
1.8662 & 0.4834
\end{array}\right], B_{2}=\left[\begin{array}{c}
-0.0238 \\
3.5141
\end{array}\right]
\end{aligned}
$$



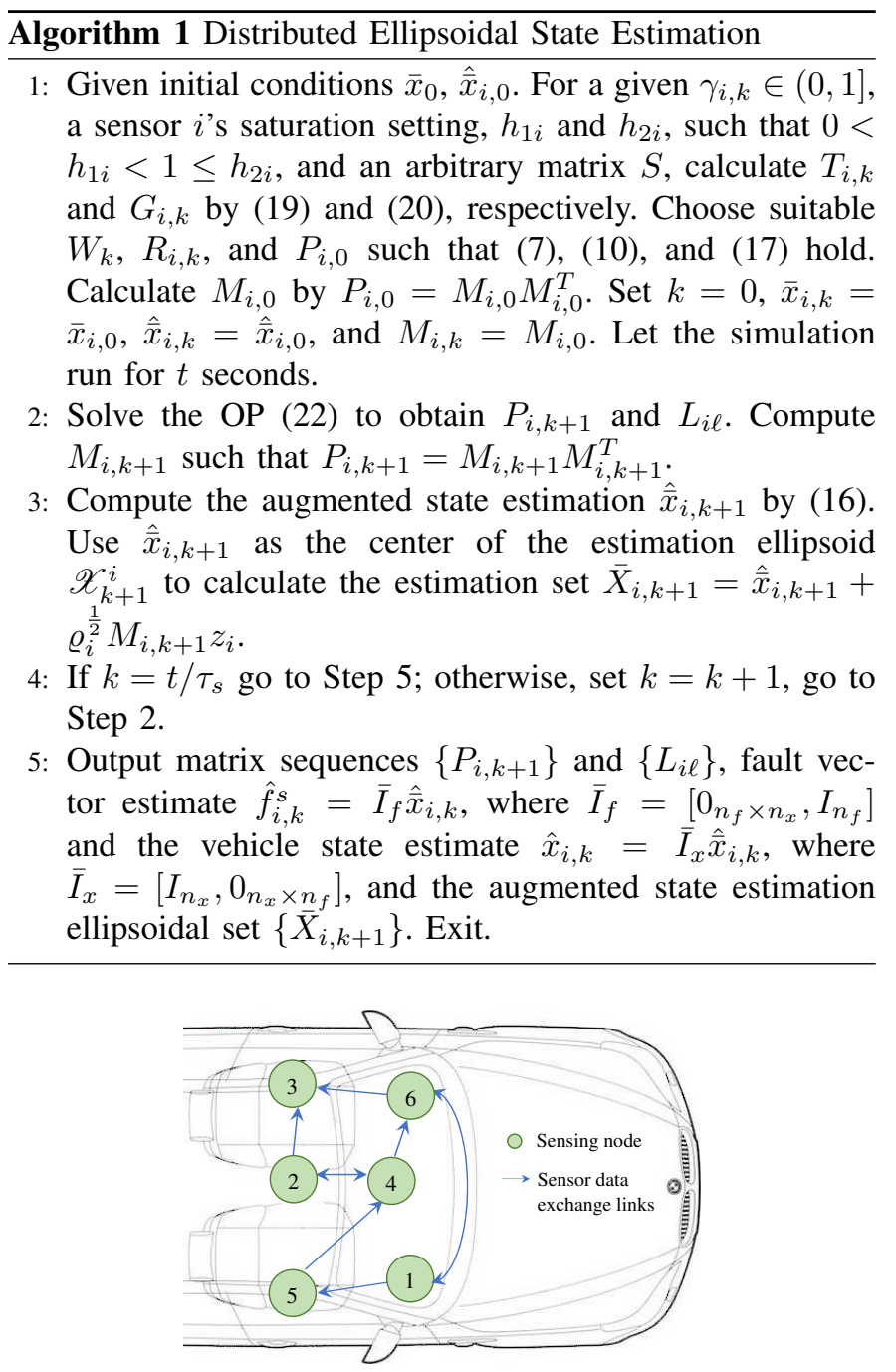

Fig. 3. Interaction topology of six sensing nodes measuring yaw rate

$$
\begin{aligned}
& A_{3}=\left[\begin{array}{cc}
0.5288 & -0.0469 \\
1.4910 & 0.3583
\end{array}\right], B_{3}=\left[\begin{array}{l}
0.0397 \\
3.1750
\end{array}\right] \\
& A_{4}=\left[\begin{array}{cc}
0.5325 & -0.0447 \\
1.4946 & 0.3615
\end{array}\right], B_{4}=\left[\begin{array}{l}
0.0466 \\
3.1815
\end{array}\right]
\end{aligned}
$$

Furthermore, the corresponding weighting functions are depicted in Fig. 4.

The UBB side wind force disturbance is assumed to be $w_{k}=-1+2 \operatorname{rand}(1,1)$. Moreover, the wind force, $f_{w}=$ $1360 \mathrm{~N}$ assuming that $V_{w}=80 \mathrm{~km} / \mathrm{h}$ and $\rho_{w}=1.3 \mathrm{~kg} / \mathrm{m}^{2}$. Hence, the discretized external disturbance matrix with $l_{w}=$ $0.5 \mathrm{~m}$ for each T-S fuzzy model rule $\ell$ is obtained as

$$
D_{w, 1}=D_{w, 2}=\left[\begin{array}{l}
0.0009 \\
0.0224
\end{array}\right], D_{w, 3}=D_{w, 4}=\left[\begin{array}{l}
0.0017 \\
0.0209
\end{array}\right] \text {. }
$$

The output of each yaw rate sensor $i$ is subject to measurement noise as $v_{i, k}=\cos \left(2 \pi f_{c v}(k-1) \tau_{s}\right)$, where $f_{c v}=i$ denoting the noise frequency. The measurement matrices are given by $C_{1}=[0,3 / 25], C_{2}=[0,3 / 20], C_{3}=[0,9 / 50]$, $C_{4}=[0,1 / 5], C_{5}=[0,4 / 25], C_{6}=[0,7 / 50]$, and the measurement noise matrices are set as $D_{v, 1}=0.00873$, $D_{v, 2}=0.01047, D_{v, 3}=0.00542, D_{v, 4}=0.00698, D_{v, 5}=$ $0.00785, D_{v, 6}=0.0096$. Choosing $W_{k}=R_{i, k}=2$, one can

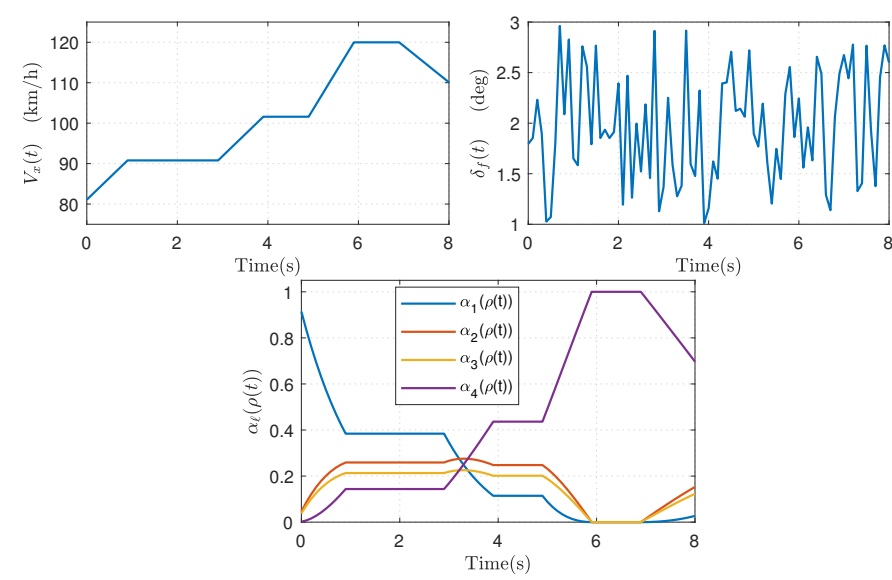

Fig. 4. Vehicle longitudinal velocity, $V_{x}$, front wheel steering angle $\delta_{f}$, and the weighting functions $\alpha_{\ell}(\rho(t)), \ell=1,2,3,4$

easily check that $w_{k}$ and $v_{i, k}$ belong to the ellipsoidal sets defined in (7) and (10), respectively.

As discussed in [15], the common technical faults in a yaw rate sensor are sensor bias and drift, which refer to constant and slow-varying errors in the measurement signal, respectively. Such failures may be caused by sensor misalignment, variation in sensor temperature, sensor circuit malfunctions, and so on [27]. To model a constant and/or a slow-varying fault, a combination of step and ramp functions is considered to represent a fault signal in each yaw rate sensor as follows:

$$
\begin{aligned}
& f_{1, k}^{s}= \begin{cases}0.1745 \operatorname{sign}\left(\sin \left(\frac{2 \pi}{90} k\right)\right), & \text { if } 20 \leq k<70 \\
0, & \text { otherwise. }\end{cases} \\
& f_{2, k}^{s}= \begin{cases}0.1745(k-10) / 10, & \text { if } 10 \leq k<20 \\
0.1745, & \text { if } 20 \leq k<50 \\
0.1745-0.1745(k-50) / 10, & \text { if } 50 \leq k<60 \\
0, & \text { otherwise. }\end{cases} \\
& f_{4, k}^{s}= \begin{cases}0.0873 \operatorname{sign}\left(\sin \left(\frac{2 \pi}{70} k\right)\right), & \text { if } 15 \leq k<65 \\
0, & \text { otherwise. }\end{cases} \\
& f_{6, k}^{s}= \begin{cases}-0.0873(k-15) / 10, & \text { if } 15 \leq k<25 \\
-0.0873, & \text { if } 25 \leq k<55 \\
-0.0873+0.0873(k-55) / 10, & \text { if } 55 \leq k<65 \\
0, & \text { otherwise. }\end{cases}
\end{aligned}
$$$$
f_{3, k}^{s}=0, f_{5, k}^{s}=0
$$

where the faults in yaw rate sensors have the maximum amplitudes $10^{\circ} / \mathrm{s}$ for $f_{1}^{s}$ and $f_{2}^{s}$, and $5^{\circ} / \mathrm{s}$ for $f_{4}^{s}$ and $f_{6}^{s}$. Also, there is no fault in yaw rate sensors 3 and 5 . Furthermore, the fault matrices are chosen as $D_{s, 1}=0.47, D_{s, 2}=0.42$, $D_{s, 3}=0.56, D_{s, 4}=0.52, D_{s, 5}=0.58$, and $D_{s, 6}=0.61$.

Considering the initial sideslip angle and yaw rate as zero, the initial augmented state is as $\bar{x}_{0}=\left[\begin{array}{lll}0, & 0, & 0\end{array}\right]^{T}$. The initial conditions of estimators are chosen as $\hat{\bar{x}}_{1,0}=$ $\left[\begin{array}{lll}0.028, & 0.168, & 0\end{array}\right]^{T}, \hat{\bar{x}}_{2,0}=\left[\begin{array}{lll}0.032, & 0.192, & 0\end{array}\right]^{T}, \hat{\bar{x}}_{3,0}=$ $\left[\begin{array}{lll}0.036, & 0.216, & 0\end{array}\right]^{T}, \hat{\bar{x}}_{4,0}=\left[\begin{array}{lll}0.023, & 0.138, & 0\end{array}\right]^{T}, \hat{\bar{x}}_{5,0}=$ $[0.033,0.198,0]^{T}$, and $\hat{\bar{x}}_{6,0}=[0.038,0.288,0]^{T}$, which define the centers of initial ellipsoidal sets (17) for each 

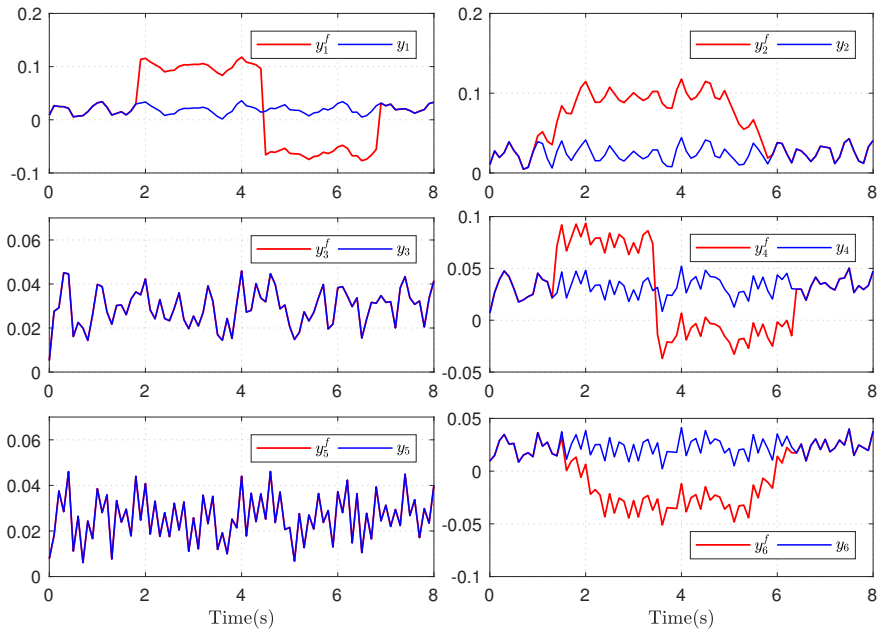

Fig. 5. Measurement output of sensor $i(\mathrm{rad} / \mathrm{s}), \forall i \in \mathcal{V}$, with fault $y_{i}^{f}$ and without fault $y_{i}$

estimator with the shape matrix and the scaling parameter taken as $P_{i, 0}=0.3 I_{3 \times 3}$ and $\varrho_{i}=1$, respectively.

The main objective is twofold: 1) To construct a bounding augmented state estimation ellipsoid for each sensing node so as to always enclose the vehicle true states, the yaw rate and the sideslip angle, and the sensor faults, at each time step, in the estimation ellipsoids of sensing nodes despite the UBB wind force disturbance and measurement noise; and 2) To perform a comparative analysis revealing the effects of the incomplete measurement and sensor saturation on the performance of the distributed joint state and sensor fault estimation process. In particular, we consider the following two different cases:

\section{A. Neither Saturated nor Incomplete Measurement (NSNIM) Case}

Let the simulation run for $8 \mathrm{~s}$. From Algorithm 1, the proposed distributed set-membership joint state and fault estimation problem is solved for an $\mathrm{AGV}$ with the presented settings. In Fig. 5, one can see that the measurement outputs of sensors $i=1,2,4,6$ have been seriously distorted by the existing faults on each sensor. However, as depicted in Fig. 6, each estimator $i$ can compensate the fault impact on the measurement output of sensor $i$ and hence, generate the vehicle yaw rate and sideslip angle estimations which accurately track their true values. Moreover, Fig. 6 shows that the true yaw rate and sideslip angle of the vehicle are always confined in the upper and lower bounds of the confidence region calculated by $\bar{I}_{x} \bar{x}_{i, k}=\bar{I}_{x}\left(\hat{\bar{x}}_{i, k}+\varrho_{i}^{\frac{1}{2}} M_{i, k} z_{i}\right)$, where $z_{i}$ is chosen as a unit sphere.

In addition to the state estimation, Fig. 7 demonstrates that estimator $i$ is able to successfully provide the estimation of the fault on sensor $i$. Also, as shown in this figure, the true value of each sensor fault is always confined in its confidence region limited with the upper bound and the lower bound computed by the proposed set-membership fault estimation technique through $\bar{I}_{f} \bar{x}_{i, k}=\bar{I}_{f}\left(\hat{\bar{x}}_{i, k}+\varrho_{i}^{\frac{1}{2}} M_{i, k} z_{i}\right)$.
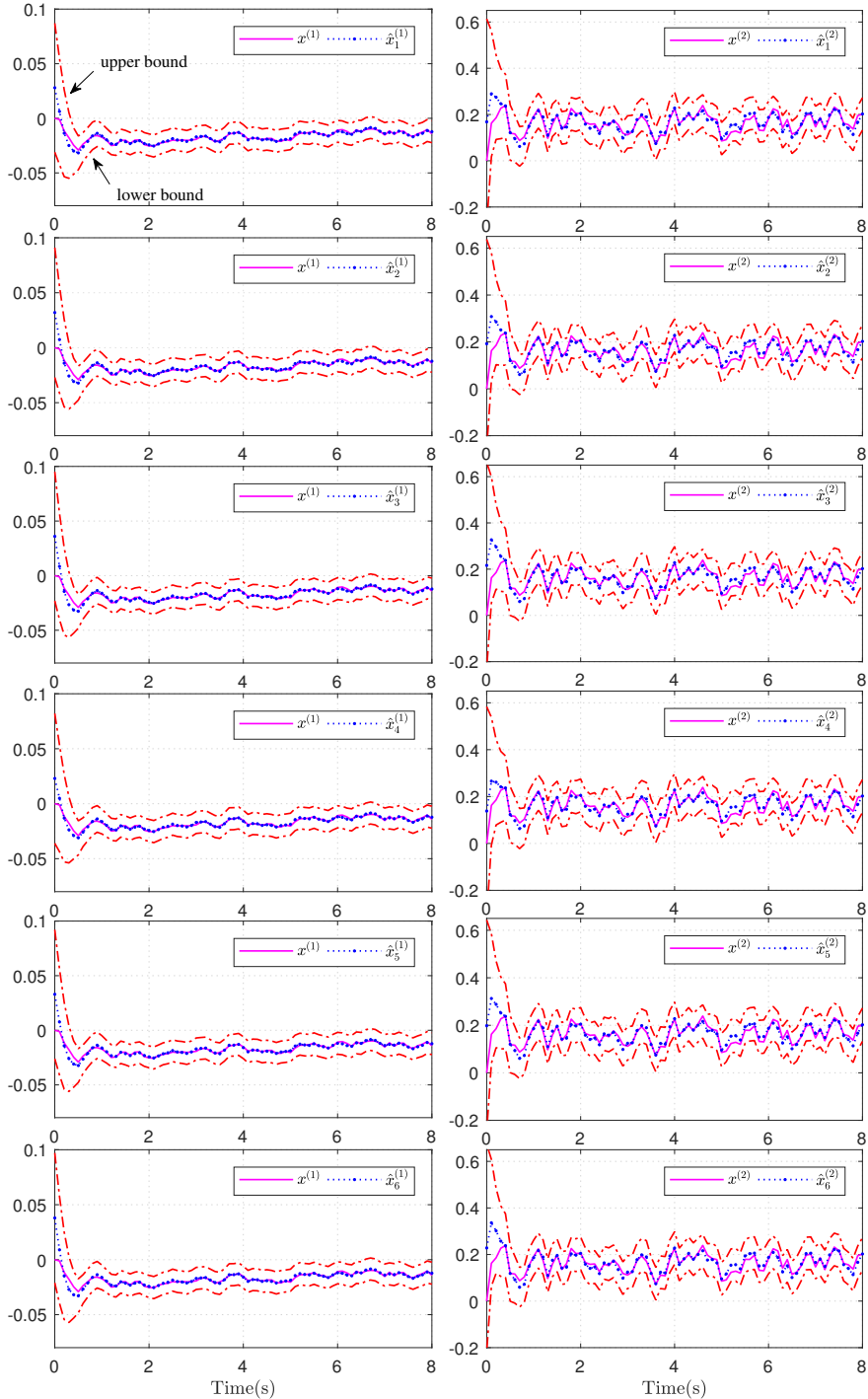

Fig. 6. True state of the vehicular sideslip angle $x^{(1)}$ (rad), yaw rate $x^{(2)}$ $(\mathrm{rad} / \mathrm{s})$, and their estimates $\hat{x}_{i}^{(1)}, \hat{x}_{i}^{(2)}$, on sensing node $i, \forall i \in \mathcal{V}$ as well as the state's upper and lower bounds

In summary, Fig. 6 and Fig. 7 confirm that the true states of the vehicle and the true value of the sensor fault are guaranteed to reside in the confidence estimated ellipsoidal set derived by each sensing node, and hence the proposed distributed set-membership augmented state estimation approach provides a guaranteed estimation ellipsoid for the AGV's state and fault in each sensor. This ellipsoidal estimation technique has great potential to enhance the stability performance of AGVs due to the fact that their implemented control units can confidently utilize the estimate sets to generate the desired control command regardless of the UBB external disturbance and measurement noise.

\section{B. Saturated and Incomplete Measurement (SAIM) Case}

In this case, it is considered that the distributed estimator $i$ may receive incomplete and saturated measurement from its neighboring yaw rate sensors due to their physical constraints, technical limitation, and accidental data loss over the signal 

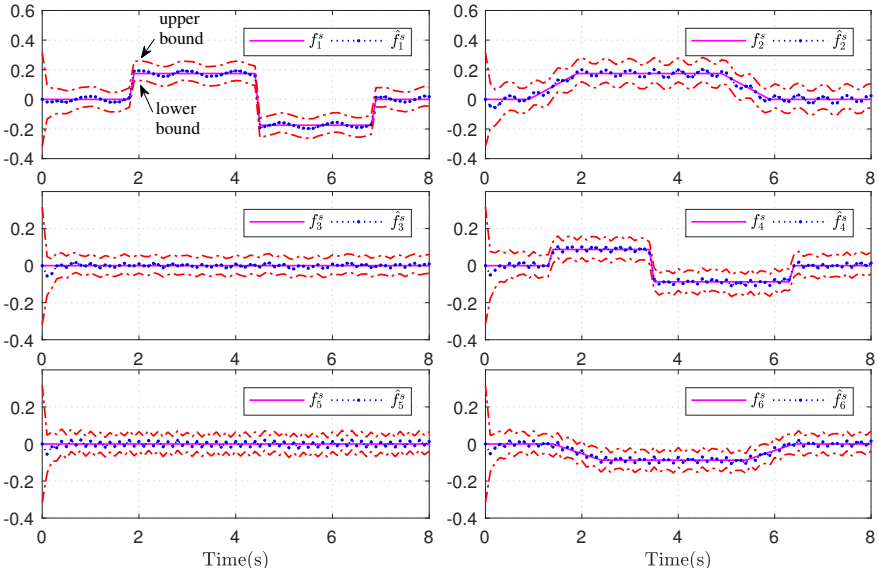

Fig. 7. Fault, $f_{i}^{s}(\mathrm{rad} / \mathrm{s})$, its estimate, $\hat{f}_{i}^{s}$, on sensing node $i, \forall i \in \mathcal{V}$, the fault's upper and lower bounds

transmission. To model the incomplete measurement of sensor $i$, the availability status of each sensor is chosen as $\gamma_{1, k} \in$ $[0.72,0.85], \gamma_{2, k} \in[0.82,0.95], \gamma_{3, k} \in[0.76,0.91], \gamma_{4, k} \in$ $[0.83,0.98], \gamma_{5, k} \in[0.78,0.90]$, and $\gamma_{6, k} \in[0.87,0.96]$. Also, the sensor saturation scalars are set as $h_{1 i}=0.8$ and $h_{2 i}=1.1 \forall i \in \mathcal{V}$, and the maximum saturation levels are chosen as $r_{1, \max }=0.08, r_{2, \max }=0.07, r_{3, \max }=0.035$, $r_{4, \max }=0.06, r_{5, \max }=0.03$, and $r_{6, \max }=0.025$.

To facilitate comparative analysis, a quadratic estimation error on each sensing node is defined as an ellipsoidal estimation performance index by $\mathcal{E}_{k+1}^{i}=e_{i, k+1}^{T} P_{i, k+1}^{-1} e_{i, k+1}$. The simulation results between the two different cases, i.e., NSNIM and SAIM, are then compared by calculating each sensing node's quadratic estimation error. In Fig. 8, one can observe that $\mathcal{E}_{k+1}^{i} \leq \varrho_{i}=1$ for each sensing node in both cases, which also verifies that the true states of the vehicle and the true value of sensor fault always reside in sensing nodes' augmented state estimation ellipsoids $\mathscr{X}_{k+1}^{i} \forall i \in \mathcal{V}$. However, it should be noted that the SAIM case provides larger quadratic estimation errors due to the impact of the incomplete measurement and the sensor saturation on the estimation performance. In other words, Fig. 8 confirms that these common practical phenomena can degrade the performance of each distributed estimator. However, the proposed set-membership estimation technique is able to adjust the bounds so as to satisfy the condition on the quadratic estimation error and hence, provides the automotive engineers with an effective methodology so that they can achieve the balance between accurate estimation performance and cost in practical vehicular applications.

\section{CONCLUSION}

The distributed set-membership joint state and sensor fault estimation problem over a multiple yaw rate sensor network configuration has been addressed for an AVG whose dynamics are under the influence of UBB external disturbance and measurement noise. Due to physical constraints, technical and communication limitations in real-world application of yaw rate sensors, it has been considered that each distributed estimator may suffer from sensor saturation and incomplete measurement signals. Distributed set-membership estimators
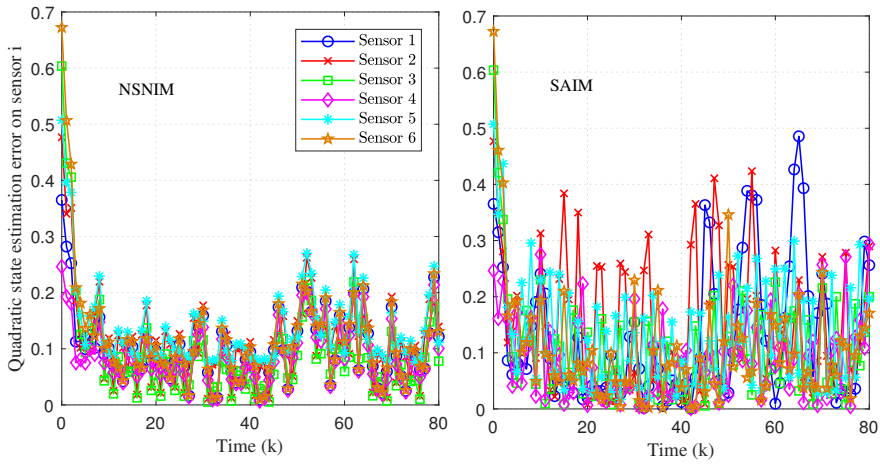

Fig. 8. Quadratic estimation error $\mathcal{E}_{k+1}^{i}$ on sensing node $i, \forall i \in \mathcal{V}$ in different cases of the NSNIM and the SAIM

have been constructed to estimate the vehicle yaw rate, sideslip angle, and each sensor's fault. The design criterion for computing the desired estimators under varying longitudinal velocity consideration has been developed to guarantee that each estimator can provide a bounding ellipsoidal estimation of the vehicle's true state and its sensor fault. To find optimized ellipsoids, a recursive convex optimization algorithm has been developed. Finally, comparative simulation results have been provided to demonstrate the effectiveness of the proposed results. As possible future research, how to develop an eventtriggered distributed state and fault estimation approach for in-vehicle networked AGVs subject to limited resources is an interesting problem. Another potential research issue is to incorporate both sensor and actuator faults and further initiate an effective distributed fault detection and isolation approach for AGVs.

\section{REFERENCES}

[1] A. Eskandarian, C. Wu, and C. Sun, "Research advances and challenges of autonomous and conntected ground vehicle," IEEE Trans. Intell. Transp. Syst., to be published, doi: 10.1109/TITS.2019.2958352.

[2] Z. Peng, J. Wang, D. Wang, and Q.-L. Han, "An overview of recent advances in coordinated control of multiple autonomous surface vehicles," IEEE Trans. Ind. Informat., vol. 17, no. 2, pp. 732-745, Feb. 2021.

[3] A. Y. S. Lam, Y.-W. Leung, and X. Chu, "Autonomous-vehicle public trasportation system: Scheduling and admission control," IEEE Trans. Intell. Transp. Syst., vol. 17, no. 5, pp. 1210-1226, May 2016.

[4] J. Ni, J. Hu, and C. Xiang, "Envelope control for four-wheel independently actuated autonomous ground vehicle through AFS/DYC integrated control," IEEE Trans. Veh. Technol., vol. 66, no. 1, pp. 97129726, Nov. 2017.

[5] E. Mousavinejad, Q.-L. Han, F. Yang, Y. Zhu, and L. Vlacic, "Integrated control of ground vehicles dynamics via advanced terminal sliding mode control," Vehicle Syst. Dyn., vol. 55, no. 2, pp. 268-294, 2017.

[6] S. A. Mortazavizadeh, A. Ghaderi, M. Ebrahimi, and M. Hajian, "Recent developments in the vehicle steer-by-wire system," IEEE Trans. Transport. Electrific., to be published, doi: 10.1109/TTE.2020.3004694.

[7] C. Huang, F. Naghdy, H. Du, and H. Huang, "Shared control of highly automated vehicles using steer-by-wire systems," IEEE/CAA J. Automatica Sinica, vol. 6, no. 2, pp. 410-423, Mar. 2019.

[8] D. Piyabongkarn, R. Rajamani, J. A. Grogg, and J. Y. Lew, "Development and experimental evaluation of a slip angle estimator for vehicle stability control," IEEE Trans. Control Syst. Technol., vol. 17, no. 1, pp. 78-88, Jan. 2009.

[9] M. Doumiati, A. C. Victorino, A. Charara, and D. Lechner, "Onboard real-time estimation of vehicle lateral tire-road forces and sideslip angle," IEEE/ASME Trans. Mechatronics, vol. 16, no. 4, pp. 601-614, Aug. 2011. 
[10] J.-H. Yoon and H. Peng, "Robust vehicle sideslip angle estimation through a disturbance rejection filter that integrates a magnetometer with GPS," IEEE Trans. Intell. Transp. Syst., vol. 15, no. 1, pp. 191-204, Feb. 2014.

[11] H. Zhang, X. Zhang, and J. Wang, "Robust gain-scheduling energyto-peak control of vehicle lateral dynamics stabilisation," Vehicle Syst. Dyn., vol. 52, no. 3, pp. 309-340, 2014.

[12] H. Zhang, G. Zhang, and J. Wang, "Sideslip angle estimation of an electric ground vehicle via finite-frequency $H_{\infty}$ approach," IEEE Trans. Transport. Electrific., vol. 2, no. 2, pp. 200-209, Jun. 2016.

[13] Y. Wang, H. R. Karimi, H.-K. Lam, and H. Yan, "Fuzzy output tracking control and filtering for nonlinear discrete-time descriptor systems under unreliable communication links," IEEE Trans. Cybern., vol. 50, no. 6, pp. 2369-2379, Jun. 2020.

[14] W. E. Lay and P. W. Lett "Wind effects on car stability," SAE Transactions, vol. 61, pp. 608-622, 1953.

[15] S. Ding, Model-Based Fault Diagnosis Techniques: Design Schemes, Algorithms, and Tools. Berlin, Germany: Springer-Verlag, 2008.

[16] R. Rajamani, Vehicle Dynamics and Control. New York, NY, USA: Springer-Verlag, 2006.

[17] A. B. Kurzhanski and I. Valyi, Ellipsoidal Calculus for Estimation and Control, 1st ed. Boston, MA, USA: Birkhäuser, 1997.

[18] F. C. Schweppe, "Recursive state estimation: Unknown but bounded errors and system inputs," IEEE Trans. Autom. Control, vol. AC-13, no. 1, pp. 22-28, Feb. 1968.

[19] X. Ge, Q.-L. Han, and F. Yang, "Event-based set-membership leaderfollowing consensus of networked multi-agent systems subject to limited communication resources and unknown-but-bounded noise," IEEE Trans. Ind. Electron., vol. 64, no. 6, pp. 5045-5054, Jun. 2017.

[20] L. Orihuela, S. Roshany-Yamchi, R. García, and P. Millán, "Distributed set-membership observers for interconnected multi-rate systems," Automatica, vol. 85, pp. 221-226, Nov. 2017.

[21] Z. Wang, X. Shen, and Y. Zhu, "Ellipsoidal fusion estimation for multisensor dynamic systems with bounded noises," IEEE Trans. Autom. Control, vol. 64, no. 11, pp. 4725-4732, Nov. 2019

[22] E. Mousavinejad, F. Yang, Q.-L. Han, X. Ge, and L. Vlacic, "Distributed cyber attacks detection and recovery mechanism for vehicle platooning," IEEE Trans. Intell. Transp. Syst., vol. 21 , no. 9, pp. 3821-3834, Sep. 2020.

[23] M. Zhou, Z. Cao, M. Zhou, J. Wang, and Z. Wang, "Zonotoptic fault estimation for discrete-time LPV systems with bounded parametric uncertainty," IEEE Trans. Intell. Transp. Syst., vol. 21 , no. 2, pp. 690700, Feb. 2020.

[24] X. Ge, Q.-L. Han, and Z. Wang, "A dynamic event-triggered transmission scheme for distributed set-membership estimation over wireless sensor networks," IEEE Trans. Cybern., vol. 49, no. 1, pp. 171-183, Jan. 2019.

[25] D. Ding, Z. Wang, and Q.-L. Han, "A set-membership approach to eventtriggered filtering for general nonlinear systems over sensor networks," IEEE Trans. Autom. Control, vol. 65, no. 4, pp. 1792-1799, Apr. 2020.

[26] H. Guo, D. Cao, H. Chen, C. Lv, H. Wang, and S. Yang, "Vehicle dynamic state estimation: State of the art schemes and prespectives," IEEE/CAA J. Automatica Sinica, vol. 5, no. 2, pp. 418-431, Mar. 2018.

[27] G. Zhang, Z. Yu, and J. Wang, "Correction of contaminated yaw rate signal and estimation of sensor bias for an electric vehicle under normal driving conditions," Mech. Syst. Signal Process., vol. 87, pp. 64-80, 2017.

[28] Z. Gao, C. Cecati, and S. X. Ding, "A survey of fault diagnosis and fault-tolerant techniques - part I: Fault diagnosis with model-based and signal-based approaches," IEEE Trans. Ind. Electron., vol. 62, no. 6, pp. 3757-3767, Jun. 2015.

[29] X. Bu, H. Dong, Z. Wang, and H. Liu, "Non-fragile distributed fault estimation for a class of nonlinear time-varying systems over sensor networks: the finite-horizon case," IEEE Trans. Signal Inf. Process. Netw., vol. 5, no. 1, pp. 61-69, Mar. 2019.

[30] K. Zhang, B. Jiang, S. X. Ding, and D. Zhou, "Robust asymptotic fault estimation of discrete-time interconnected systems with sensor faults," IEEE Trans. Cybern., to be published, doi: 10.1109/TCYB.2020.2986386.

[31] S. Mammar, S. Glaser, and M. Netto, "Vehicle lateral dynamics estimation using unknown input proportional-integral observers," in Proc. Amer. Control Conf., Minneapolis, MN, Jun. 2006, pp. 4658-4663.

[32] Z. Gao, "Fault estimation and fault-tolerant control for discrete-time dynamic systems," IEEE Trans. Ind. Electron., vol. 62, no. 6, pp. 3874 3884, Jun. 2015.
[33] L. Ma, Z. Wang, H.-K. Lam, and N. Kyriakoulis, "Distributed eventbased set-membership filtering for a class of nonlinear systems with sensor saturations over sensor networks," IEEE Trans. Cybern., vol. 47, no. 11, pp. 3772-3783, Nov. 2017.

[34] X. Ge, Q.-L. Han, M. Zhong, and X.-M. Zhang, "Distributed Krein space-based attack detection over sensor networks under deception attacks," Automatica, vol. 109, art. no. 108557, Nov. 2019.

[35] S. Liu, Z. Wang, G. Wei, and M. Li, "Distributed set-membership filtering for multirate systems under the round-robin scheduling over sensor networks," IEEE Trans. Cybern., vol. 50, no. 5, pp. 1910-1920, May 2020.

[36] N. Alyazidi and M. Mahmoud, "Distributed $H_{2} / H_{\infty}$ filter design for discrete-time switched systems," IEEE/CAA J. Automatica Sinica, vol. 7, no. 1, pp. 158-168, Jan. 2020.

[37] Y. Zhang, L. Du, and F. Lewis, "Stochastic DoS attack allocation against collaborative estimation in sensor networks," IEEE/CAA J. Automatica Sinica, vol. 7, no. 5, pp. 1225-1234, Sep. 2020.

[38] X. Ge, Q.-L. Han, X.-M. Zhang, L. Ding, and F. Yang, "Distributed event-triggered estimation over sensor networks: A survey," IEEE Trans. Cybern., vol. 50, no. 3, pp. 1306-1320, Mar. 2020.

[39] L. Ghaoui and G. Calafiore, "Robust filtering for discrete-time systems with bounded noise and parameteric uncertainty," IEEE Trans. Autom. Control, vol. 46, no. 7, pp. 1084-1089, Jul. 2001.

[40] H. Dahmani, M. chadli, A. Rabhi, and A. El Hajjaji, "Vehicle dynamic estimation with road bank angle consideration for rollover detection: theoretical and experimental studies," Vehicle Syst. Dyn., vol. 51, no. 12, pp. 1853-1871, 2013.

[41] K. Tanaka, H. Wang, Fuzzy Control Systems Design and Analysis: A Linear Matrix Inequality Approach. New York, NY, USA: WileyInterscience, 2001.

[42] J. Lu and T. A. Brown, "Vehicle side slip angle estimation using dynamic blending and considering vehicle attitude information," United States Patent 6671595B2, Dec. 30, 2003.

[43] G. Wei, S. Liu, Y. Song, and Y. Liu, "Probability-guaranteed setmembership filtering for systems with incomplete measurements," $\mathrm{Au}$ tomatica, vol. 60 , pp. 12-16, Oct. 2015.

[44] X.-M. Zhang, Q.-L. Han, X. Ge, D. Ding, L. Ding, D. Yue, and C. Peng, "Networked control systems: A survey of trends and techniques," IEEE/CAA J. Automatica Sinica, vol. 7, no. 1, pp. 1-17, Jan. 2020

[45] A. Ben-Israel and T. N. E. Greville, Generalized Inverses: Theory and Applications, 2nd ed. NY, USA: Springer, 2003.

[46] R. E. Skelton, T. Iwasaki, and K. Grigoriadis, A Unified Algebraic Approach to Linear Control Design. Bristol, PA: Taylor \& Francis, 1998.

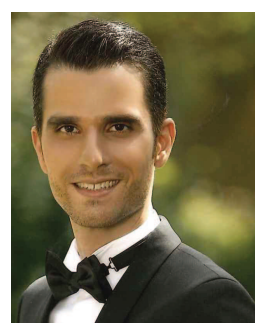

Eman Mousavinejad (S'14-M'19) received the B.Sc. degree in mechanical engineering from Azad University, Tehran, Iran, in 2006, the M.Sc. degree in mechatronics (control engineering) from the Sharif University of Technology, Tehran, Iran, in 2009, the M.Phil. and Ph.D. degrees in mechatronics (control engineering) from Griffith University, Gold Coast, QLD, Australia, in 2016 and 2020, respectively.

Dr. Mousavinejad is currently a Research Associate with the School of Electrical and Information Engineering, the University of Sydney, Sydney, NSW, Australia. He is also a Visiting Research Fellow with the School of Engineering and Built Environment, Griffith University. His current research interests include secure, intelligent, and distributed control and estimation, reinforcement learning, and cyber-physical systems.

Dr. Mousavinejad was a recipient of the Award of Excellence in a Research Thesis, Griffith University in 2020. 


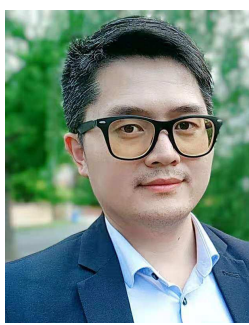

Xiaohua Ge (M'18-SM'21) received the B.Eng. degree in electronics and information engineering from Nanchang Hangkong University, Nanchang, China, in 2008, the M.Eng. degree in control theory and control engineering from Hangzhou Dianzi University, Hangzhou, China, in 2011, and the Ph.D. degree in computer engineering from Central Queensland University, Rockhampton, QLD, Australia, in 2014.

From 2011 to 2013, he was a Research Assistant with the Centre for Intelligent and Networked Systems, Central Queensland University, where he was a Research Fellow in 2014. From 2015 to 2017, he was a Research Fellow with the Griffith School of Engineering, Griffith University, Gold Coast, QLD, Australia. He is currently a Senior Lecturer with the School of Software and Electrical Engineering, Swinburne University of Technology, Melbourne, VIC, Australia. His research interests include networked, event-triggered, secure, and intelligent control and estimation, and their applications in autonomous vehicles and connected vehicles.

Dr. Ge is a Highly Cited Researcher according to Clarivate Analytics. He received The 2019 IEEE Systems, Man, and Cybernetics Society Andrew P. Sage Best Transactions Paper Award and The 2017 IEEE Transactions on Cybernetics Outstanding Reviewer Award. He is an Associate Editor of the IEEE TRANSACTION ON SYSTEMS, MAN, AND CYBERNETICS: SYSTEMS, and was a Guest Editor for several Special Issues.

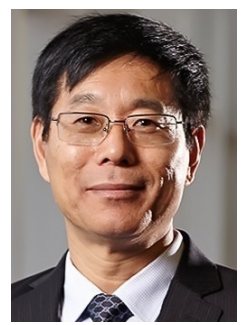

Qing-Long Han (M'09-SM'13-F'19) received the B.Sc. degree in Mathematics from Shandong Normal University, Jinan, China, in 1983, and the M.Sc. and $\mathrm{Ph} . \mathrm{D}$. degrees in Control Engineering from East China University of Science and Technology, Shanghai, China, in 1992 and 1997, respectively.

Professor Han is Pro Vice-Chancellor (Research Quality) and a Distinguished Professor at Swinburne University of Technology, Melbourne, Australia. He held various academic and management positions at Griffith University and Central Queensland University, Australia. His research interests include networked control systems, multi-agent systems, time-delay systems, smart grids, unmanned surface vehicles, and neural networks.

Professor Han was a Highly Cited Researcher in both Engineering and Computer Science (Clarivate Analytics, 2019-2020). He was one of Australia's Top 5 Lifetime Achievers (Research Superstars) in Engineering and Computer Science (The Australian's 2020 Research Magazine). He is currently one of Australia's Top 4 Researchers in Computer Science and Electronics (Guide2Research). He was the recipient of The 2020 IEEE Systems, Man, and Cybernetics (SMC) Society Andrew P. Sage Best Transactions Paper Award, The 2020 IEEE Transactions on Industrial Informatics Outstanding Paper Award, and The 2019 IEEE SMC Society Andrew P. Sage Best Transactions Paper Award.

Professor Han is a Fellow of The Institution of Engineers Australia. $\mathrm{He}$ has served as an AdCom Member of IEEE Industrial Electronics Society (IES), a Member of IEEE IES Fellow Committee, and Chair of IEEE IES Technical Committee on Networked Control Systems. He has acted as CoEditor for Australian Journal of Electrical \& Electronics Engineering, an Associate Editor for 12 international journals, including the IEEE TRANSACTIONS ON CYBERNETICS, the IEEE TRANSACTIONS ON INDUSTRIAL INFORMATICS, IEEE INDUSTRIAL ELECTRONICS MAGAZINE, the IEEE/CAA JOURNAL OF AUTOMATICA SINICA, Control Engineering Practice, and Information Sciences, and a Guest Editor for 13 Special Issues.

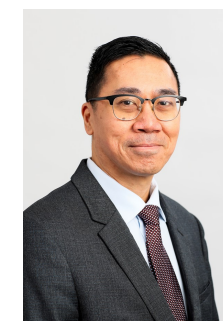

Teng Joon (T. J.) Lim (S'92-M'95-SM'02-F'17) obtained the B.Eng. degree in Electrical Engineering with first-class honours from the National University of Singapore (NUS) in 1992, and the Ph.D. degree from the University of Cambridge in 1996.

From September 1995 to November 2000, he was a researcher at the Centre for Wireless Communications in Singapore, one of the predecessors of the Institute for Infocomm Research (I2R). From December 2000 to May 2011, he was Assistant Professor, Associate Professor, then Professor at the University of Toronto's Edward S. Rogers Sr. Department of Electrical and Computer Engineering. From June 2011 to January 2020, he was a Professor at the Electrical \& Computer Engineering Department of NUS, where he served as a Deputy Head from July 2014 to August 2015. From September 2015 through December 2019, he served as Vice-Dean (Graduate Programs) in the NUS Faculty of Engineering. Since January 2020, he has served as Deputy Dean and Associate Dean (Education) at the Faculty of Engineering in the University of Sydney.

Professor Lim is an Associate Editor for IEEE Potentials, was an Area Editor of the IEEE Transactions on Wireless Communications from September 2013 to September 2018, and previously served as an Associate Editor for the same journal. He has also served as an Associate Editor for IEEE Wireless Communications Letters, Wiley Transactions on Emerging Telecommunications Technologies (ETT), IEEE Signal Processing Letters and IEEE Transactions on Vehicular Technology. He has volunteered on the organizing committee of a number of IEEE conferences, including serving as the TPC co-chair of IEEE Globecom 2017. He chaired the Singapore chapter of the IEEE Communications Society in 2017 and 2018, and was a Distinguished Lecturer of the IEEE Vehicular Technology Society for 2019-20. He was also a co-winner of the IEEE Communications Society's 2020 Heinrich Hertz Award for Best Communication Letter.

Professor Lim's research interests span many topics within wireless communications, including cyber-security in the Internet of Things, heterogeneous networks, cooperative transmission, energy-optimized communication networks, multi-carrier modulation, MIMO, cooperative diversity, cognitive radio, and stochastic geometry for wireless networks, and he has published widely in these areas.

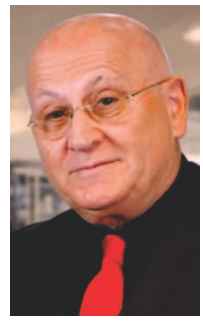

Ljubo Vlacic (M'92-SM'96) received the Graduate Diploma degree in electrical engineering and the M.Phil. and Ph.D. degrees in control systems engineering from the University of Sarajevo, Sarajevo, Bosnia and Herzegovina, in 1973, 1976, and 1986, respectively. He also graduated Conservatorium of Music (Violin), the University of Sarajevo in 1976.

Professor Emeritus Vlacic is a Control Systems Scientist and a Practitioner, renowned for his contributions to co-operative driverless vehicles and intelligent control systems research and development. $\mathrm{He}$ has held a number of leading roles in both industry and academia. $\mathrm{He}$ is currently with the Institute for Integrated and Intelligent Systems, Griffith University, Queensland, Australia.

Professor Emeritus Vlacic is a recipient of 22 awards, including the IEE Achievement Medal (world-wide), the Sir Lionel Hooke Award, the Queensland Professional Engineer of the Year Award, the Gold Coast Business Events Ambassador Award, etc. He organised and hosted twelve national and international scientific conferences. His research achievements made news headlines and were broadcast through media outlets throughout the world.

Professor Emeritus Vlacic is currently the Editor-in-Chief of the IEEE Intelligent Transportation Systems Magazine, Chair of the IET Queensland Local Network and Chair of the IET Australia Forum. He played violin with professional symphony orchestra. 